\title{
Effective diffusivity in partially-saturated carbon-fiber gas diffusion layers: Effect of local saturation and application to macroscopic continuum models
}

\author{
Pablo A. García-Salaberri ${ }^{1}$, Jeff T. Gostick ${ }^{2}$, Gisuk Hwang ${ }^{3}$, Adam Z. Weber ${ }^{4}$ \\ and Marcos Vera ${ }^{1, \mathrm{~A}}$ \\ ${ }^{1}$ Dept. de Ingeniería Térmica y de Fluidos, Universidad Carlos III de Madrid, Leganés, \\ 28911, Spain \\ ${ }^{2}$ Dept. of Chemical Engineering, McGill University, Montreal, QC, Canada \\ ${ }^{3}$ Dept. of Mechanical Engineering, Wichita State University, Wichita, KS, USA \\ ${ }^{4}$ Environmental Energy Technologies Division, Lawrence Berkeley National Laboratory, \\ Berkeley, CA, USA \\ A Corresponding author: Tel: +34 916249987 Fax: +34 916249430 \\ E-mail: mvcoello@ing.uc3m.es \\ URL: http://fluidosuc3m.es/people/mvcoello
}




\begin{abstract}
Macroscopic continuum models are an essential tool to understand the complex transport phenomena that take place in gas diffusion layers (GDLs) used in polymer electrolyte fuel cells (PEFCs). Previous work has shown that macroscopic models require effective properties obtained under uniform saturation conditions to get a consistent physical formulation. This issue, mostly unappreciated in the open literature, is addressed in detail in this work. To this end, lattice Boltzmann simulations were performed on tomographic images of dry and water-invaded carbon-paper GDL subsamples with nearly uniform porosity and saturation. The computed effective diffusivity shows an anisotropic dependence on local porosity similar to that reported for morphologically analogous GDLs. In contrast, the dependence on local saturation is rather isotropic, following a nearly quadratic power law. The capability of the local correlations to recover the layer-scale properties obtained from inhomogeneous GDLs is checked by global averaging. Good agreement is found between the upscaled results and the diffusivity data of the GDL from which the present subdomains were taken, as well as other global data presented in the literature. A higher blockage effect of local saturation is, however, expected for the under-the-rib region in operating PEFCs.
\end{abstract}

Keywords: gas diffusion layer; macroscopic continuum modeling; effective diffusivity; water saturation; X-ray tomography; lattice Boltzmann method 


\section{Introduction}

Polymer electrolyte fuel cells (PEFCS) are a promising technology for transportation applications due to their virtually zero emission of air pollutants, high power density, short refueling time, long range, high thermodynamic efficiency, and fast dynamic response [1]. The core structure of a PEFC is the membrane-electrode assembly (MEA), which is composed of a central proton-exchange membrane (PEM) coated with porous anode and cathode catalyst layers (CLs), and then assembled between fibrous gas diffusion layers (GDLs). The MEA is sandwiched between two bipolar plates (BPPs), which provide mechanical support to the system, supply reactants to the CLs, remove generated and condensed liquid water, and collect the output current of the cell.

Fuel-cell designers are continually striving to optimize the transport of reactants and products throughout the porous components of the MEA [2-5]. Macroscopic continuum models are an indispensable tool toward this goal, enabling a better understanding of the complex multiphase and multiphysics phenomena that take place in fuel-cell electrodes [6-9]. Mathematical modeling in PEFCs is always based on the conservation equations of mass, momentum, energy, charge, and species. A key limitation when applying such models to PEFCs, however, is the need to input constitutive relationships to describe the transport processes involved in the above set of equations. The accuracy and reliability of macroscopic models is highly sensitive to the correlations adopted for the effective properties of the porous layers comprising the MEA $[6,8,10,11]$. As a result, a large body of work on the mass, electrical, and thermal transport properties of GDLs can be found in the literature (see, e.g., [12] and references therein).

Diffusive gas transport in GDLs plays a crucial role at high current densities, when mass transport limitations are dominant due to the high reactant consumption rates and the blockage effect of condensed product water $[4,13]$. The effective gas diffusivity in partially-saturated porous media, $D^{\text {eff,wet }}$, depends on the void volume fraction, $\varepsilon_{\mathrm{v}}$, and 
the so-called tortuosity, $\tau$, which accounts for all the structural characteristics (size, morphology, topology, etc.) of gas transport pathways [14-16]. It is typically normalized by the bulk diffusion coefficient, $D_{\mathrm{bulk}}$, in the open space:

$$
\frac{D^{\text {eff,wet }}}{D_{\text {bulk }}}=\frac{\varepsilon_{\mathrm{v}}}{\tau}=\frac{\varepsilon(1-s)}{\tau}
$$

where $\varepsilon$ and $s$ are the porosity and water saturation of the porous material, respectively. The above expression can be decomposed into two separate functions in order to differentiate the influence of the dry porous medium from that of liquid water on the effective diffusivity $[15,17-19]$ :

$$
\frac{D^{\text {eff,wet }}}{D_{\text {bulk }}}=\frac{D^{\text {eff,dry }}}{D_{\text {bulk }}} \frac{D^{\text {eff,wet }}}{D^{\text {eff,dry }}}=f(\varepsilon) g(s)
$$

The normalized function $f(\varepsilon)=D^{\text {eff,dry }} / D_{\text {bulk }}$ measures the effect of the diffusive resistance of the dry material as a function of porosity, while the normalized function $g(s)=D^{\text {eff,wet }} / D^{\text {eff,dry }}$ accounts for the relative reduction due to the presence of water saturation. Both components are usually characterized by power-like relationships. A wider variety of correlations is adopted for $f(\varepsilon)$, which include or not the percolation threshold of carbon-fiber materials $[4,16,17,20-22]$, whereas the impact of water is almost universally fitted by a power law of the form [4,15,17-19]:

$$
g=(1-s)^{\mathrm{n}}
$$

where the exponent $\mathrm{n}$ characterizes the blockage effect of water saturation, and is determined from suitable experimental or computational data. With this form for $g(s)$, the dry effective diffusivity, $D^{\text {eff,dry, }}$ is recovered for unsaturated conditions $(s=0)$. A detailed review on the normalized functions $f(\varepsilon)$ and $g(s)$ for carbon-paper GDLs can be found in the precursor to this work [15].

It has been a common practice to correlate the effective diffusivity in terms of global variables, namely, average porosity, $\varepsilon_{\text {avg, }}$ and average saturation, $s_{\text {avg, }}$, without considering the spatial distribution of water inside the GDL. However, several groups have recently pointed out that the GDL effective diffusivity is not uniquely described by 
average (or total) saturation, but rather that the saturation distribution has major effects [15,23-25]. This finding has a serious implication on fuel-cell modeling. Macroscopic continuum models implicitly assume in their construction that every computational node composing the GDL is homogenous $[8,17,26]$, i.e., each node has a single associated value of saturation and negligible saturation distribution within it. Therefore, correlations for continuum models should be estimated from samples that also have flat (i.e., uniform) saturation profiles; it is inconsistent to evaluate the diffusive fluxes between the numerical nodes in a GDL model (an inherently local scale) using data obtained from GDLs with non-uniform saturation distributions (an inherently global effect).

This issue is illustrated graphically in Figure 1. Consider the six experimental effective diffusivity measurements shown in Figure $1(\mathrm{a})$. Experiments $A, B$ \& $C$ were conducted on samples with nearly uniform saturation, while experiments $D, E \& F$ were conducted on samples with significant saturation gradients. It has been recently shown [15] that the through-plane diffusivity measured in $D, E \& F$ will be much lower than in $A, B \& C$ due to the strong bottleneck effect induced by the high-saturation regions, even if the average saturations are the same. This is represented in Figure $1(a)$ by the plot showing the

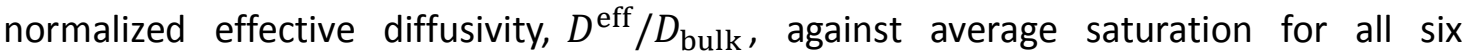
experiments. Now consider the modeled GDL domain shown in Figure 1(b). Such a macroscopic model predicts the local saturation at every computational node as a part of the numerical solution $[8,17,26]$, which by definition is a single locally-uniform value. Modeling the effective diffusivity through each individual node requires a constitutive relationship that is representative of the local conditions in that homogenous node; namely, it requires data taken from experiments $A, B$ \& $C$, and not from $D, E \& F[17,27]$. The vast majority of attempts to provide functional relationships for the GDL effective diffusivity, however, have done so using experiments similar to $D, E \& F[18,28-30]$. The main reason for this is that a saturation distribution of some sort will always exist in any realistic experiment, such as a saturation gradient for water invasion or isolated clusters 
for water withdrawal. In the present work, a procedure for estimating local correlations for the effective diffusivity vs. porosity and saturation in carbon-paper GDLs is presented. Diffusive gas transport at the sub-pore scale is simulated by the lattice Boltzmann method (LBM) on X-ray computed tomography (XCT) images of GDL subsamples with nearly uniform porosity and saturation. The methodology reported here could be applied to other transport properties as well, such as the permeability [31,32] or thermal conductivity [33], both of which are well within the capabilities of the LBM [15]. The ability of the LBM to easily manage complex geometries has been leveraged extensively to study the transport properties of fuel-cell components under dry conditions $[15,19,34-50]$, as well as wet conditions considering simulated water distributions [49-53], and XCT images of water-invasion experiments [15] or running fuel cells [19].

The organization of the paper is as follows. In Section 2, the main aspects of the waterinvasion experiments, the LBM model, and the criteria used for the selection of the GDL subsamples are presented. The numerical results are discussed in Section 3, which is further divided in three subsections. The computed results for the dry and wet local effective diffusivities are presented in Sections 3.1 and 3.2, respectively. Due to the anisotropy of carbon-paper GDLs, the diffusive properties in both the through- and inplane directions of the material are examined separately. In Section 3.3, the capability of the local results to recover the global (i.e., layer-scale) effective properties reported in our previous work [15] and other studies in the field [18,19] is explored by global averaging. Finally, some concluding remarks and recommendations are given in Section 4. 


\section{Methods}

\subsection{Water Invasion Experiments}

Water-invasion experiments were conducted on an uncompressed $10 \%$ by weight PTFEtreated Toray ${ }^{\circledR}$ TGP-H-120 GDL (Fuel Cell Earth LLC, USA) at Lawrence Berkeley National Lab's Advanced Light Source (ALS) synchrotron (Beamline 8.3.2). Water was injected into the sample at different applied capillary pressures by varying the static head of water in a tube connected to the bottom of the sample, while air was allowed to escape out the top face through a PTFE membrane. The hydrophobic membrane prevented liquid water from leaving the system, thereby enabling saturations above the breakthrough point to be reached. Capillary pressure, $p_{\mathrm{c}}=p_{\mathrm{l}}-p_{\mathrm{g}}$, was raised from 1 to $8 \mathrm{kPa}$ in $1 \mathrm{kPa}$ increments, so that the porous medium varied from very low $\left(s_{\mathrm{avg}} \approx 0.01\right)$ to almost fully-saturated $\left(s_{\mathrm{avg}} \approx 0.9\right.$ ) conditions. X-ray tomography images were taken at each capillary pressure with a resolution of $1.33^{3} \mu \mathrm{m}^{3} /$ voxel. Only one sample was tested, so the impact of material variability cannot be ascertained. Nevertheless, the size of the sample used in this work was large enough $(1.3 \times 1.3 \times 0.28 \mathrm{~mm})$ so as to evaluate the $\mathrm{GDL}$ effective properties in a large, representative number of random subdomains at all capillary pressures $\left(\sim 2,500\right.$ simulations). The commercial software Octopus ${ }^{\circledR} 8.3$ was used to filter and reconstruct the images of the scanned materials. The reconstructed 3D image stacks were then segmented into the three phases composing the porous media, i.e., GDL solid structure (fibers, binder, and PTFE), air, and water. The code for the segmentation of the image stacks was implemented in MATLAB ${ }^{\circledR}$, relying on the image processing software Fiji for some standard operations. Additional details on the experimental set-up, the reconstruction of the image stacks, and the segmentation algorithm were recently presented in [15].

\subsection{Lattice Boltzmann Model}

Diffusive gas transport in the GDL was simulated at the sub-pore scale using the LBM 
$[54,55]$. The numerical model was implemented in the parallel open-source LB solver Palabos [56]. A 3D seven-speed D3Q7 single relaxation time [57] model was used for the simulations. The system was excited by imposing a concentration difference between 1 and 0 at the outermost faces in the direction under study according to the formulation of Zou \& He [58]. On the remaining faces of the computational domain, as well as the interfacial solid and water voxels (i.e., nodes) of the GDL, no-flux boundary conditions were prescribed using a half-way bounce back scheme [59]. In this way, solid and water phases were both treated as non-conducting to gas transport. The normalized effective gas diffusivity of the porous media, $D_{\mathrm{j}}^{\text {eff }} / D_{\mathrm{bulk}}$, was computed as the ratio of the overall flux in the direction (j) of interest to the bulk flux in that direction:

$$
\frac{D_{\mathrm{j}}^{\text {eff }}}{D_{\text {bulk }}}=\frac{\iint_{A} j_{\mathrm{j}} \mathrm{dA}}{A\left(D_{\text {bulk }} \Delta C_{\mathrm{j}} / L_{\mathrm{j}}\right)}
$$

where $A$ is the cross-sectional area of the examined GDL domain to the j-direction under study, and $j_{\mathrm{j}}, \Delta C_{\mathrm{j}}=1$, and $L_{\mathrm{j}}$ are the local diffusive flux in a computational node, the prescribed concentration difference, and the length of the GDL domain along j-direction, respectively. Simulations were run until steady state on the supercomputers Gullimin and Colosse, managed by Calcul Québec and Compute Canada [60]. Further details on the formulation and implementation of the numerical model can be found in [15].

\subsection{Selection of Subdomains}

A massive computational campaign including more than 2,500 simulations was carried out to determine the local effective diffusivity of the GDL under dry and partiallysaturated conditions for both the through- and in-plane directions. As depicted in Figure 2(a), individual computations were performed on arbitrary but representative and uniform subdomains of variable size extracted from a larger domain of $1.3 \times 1.3 \times 0.28 \mathrm{~mm}$ centered on the injection tube.

In principle, effective transport properties to be used in macroscopic continuum models 
should be determined from representative elementary volumes (REVs) $[26,61]$. By definition, a REV is the smallest subset of a material that displays the same macroscopic (i.e., volume-averaged) properties (porosity, saturation, effective diffusivity, etc.) as larger subsets. A REV must therefore satisfy two requirements: (i) it has to be large enough to contain a representative volumetric average of the discrete microstructure of the material; and (ii) it has to be small enough to not be affected by any macroscopic inhomogeneity of the porous medium [26]. The applicability of these criteria to the Toray ${ }^{\circledR}$ TGP-H-120 GDL was evaluated by computing the average porosity and water saturation of 20,000 random subsamples of varying thickness, $L_{z}$, while keeping the cross-sectional length in the material plane equal to $L_{\mathrm{x}}=L_{\mathrm{y}}=400 \mu \mathrm{m}$. Figure 2(b)-(c) shows the results of this analysis. As can be seen, neither porosity nor saturation show a single-valued plateau over which a REV can be defined [26]. In the case of porosity shown in Figure 2(b), the subdomains exhibit wide fluctuations for small sizes due to the microscopic granularity of the material, and a rising tail as the largest subdomains contain a significant fraction of the highly porous surface regions found in carbon-paper GDLs $[15,19,62]$. For intermediate sizes, the porosity shows a stable plateau, indicating that the subsamples are meaningful averages of the discrete material; this satisfies part (i) of the REV definition above. However, the porosity at the plateau never settles on a single value due to the heterogeneities in the core region of the GDL $[15,19,62]$, which violates requirement (ii) [26]. Figure 2(c) shows the same analysis for water saturation; but unlike porosity, there is almost no indication of a plateau at any length scale. This is expected for two-phase flow, since saturation gradients in thin GDLs are large [15], and thus the saturation level in any subdomain strongly depends on where it is located and its size [63].

Given the inapplicability of the classical REV concept to highly heterogeneous partiallysaturated finite-thickness GDLs [15], a more flexible definition was adopted here. In order to preserve the basic requirements of representativeness and uniformity for a REV [26], two constraints were imposed when selecting subdomains. The first requirement 
was that the subdomains had to be volumetrically uniform, so that the heterogeneities of porosity and saturation inside them were significantly small as compared to their average values (requirement ii). Then, among all these uniform subsamples the analysis was restricted to those that provided a representative volume average of the GDL microstructure (requirement i). This methodology enabled the estimation of functional relationships for the local effective diffusivity on local porosity and saturation required for the implementation of physically-consistent macroscopic models. The strategy used to meet these two selection criteria is described below.

To quantify the extent of variation of the porosity and saturation distributions in each GDL subdomain, the following uniformity factor, UF, was introduced:

$$
\mathrm{UF}=1-\frac{\operatorname{MAD}\left(\bar{\lambda}_{\mathrm{z}}\right)}{\lambda_{\mathrm{avg}}}
$$

where $\operatorname{MAD}\left(\bar{\lambda}_{\mathrm{z}}\right)$ is the mean absolute deviation of the porosity $\left(\bar{\lambda}_{\mathrm{z}}=\bar{\varepsilon}_{\mathrm{z}}\right)$ or saturation $\left(\bar{\lambda}_{\mathrm{z}}=\bar{s}_{\mathrm{z}}\right)$ distribution in the through-plane direction, and $\lambda_{\mathrm{avg}}$ is the average porosity $\left(\lambda_{\mathrm{avg}}=\varepsilon_{\mathrm{avg}}\right)$ or saturation $\left(\lambda_{\mathrm{avg}}=s_{\mathrm{avg}}\right)$ of the particular subdomain under study. Only subdomains with UF $\geq 0.75$ for both saturation and porosity were taken into account. No restrictions were imposed on the distributions in the material plane due to the significantly lower changes in this direction as compared to the strong saturation gradients and porosity variations found in the through-plane direction (see [15] for details).

To evaluate the representativeness of the subsamples, the chord-length distribution [43] was used to gauge the size, shape and orientation of the pore space. This technique provides an accurate scan of the pore structure of the GDL by measuring the amount and length of chords, i.e., lines with a specific orientation, that connect interfacial voxels delimiting the air phase. As illustrated in Figure 3(a), the chord-length distributions in the GDL were calculated by generating a 3D binary mask containing a square array of white lines (labeled 1 ) that spanned the GDL in one direction with a specified spacing 
between lines of one voxel in the other two orthogonal directions. This binary mask was then multiplied by the image stack where the air phase was set to white (1's) and the remaining phases to black (0's). The resulting images contained lines passing through the pore space, creating white chords (1's) with lengths corresponding to the size of the pore space. A chord-length distribution was obtained by performing a cluster labeling on the chords, and then counting the chords of each length to create a histogram. The chordlength distribution in any spatial direction was conveniently determined by rotating the image stacks prior to analysis. This procedure can be also applied to the invading water or the solid GDL structure, by simply changing the phase of interest.

The air chord-length distributions of the dry GDL in the through- and in-plane directions are shown in Figure 3(b). Based on these distributions, the extent of the subdomains in the material plane, $L_{\mathrm{x}} \times L_{y}$, was set to $400 \times 400 \mu \mathrm{m}^{2}$, thus well above the maximum air chord length in the material plane $\left(l_{\mathrm{a}, \mathrm{IP}}^{\mathrm{dry}, \max } \approx 300 \mu \mathrm{m}\right)$. The length in the through-plane direction, $L_{\mathrm{z}}$, was varied between 60 and $120 \mu \mathrm{m}$. These thicknesses were significantly larger than the average air chord length in the through-plane direction $\left(l_{\mathrm{a}, \mathrm{avg}, \mathrm{TP}}^{\mathrm{dry}} \approx\right.$ $30 \mu \mathrm{m})$, with about $90-99 \%$ of the chords being shorter than them. Such dimensions ensured that the subdomains were representative of the pore structure of the finitethickness GDL in order to evaluate the local impact of the dry porous medium, as well as the relative effect of water, on the effective gas diffusivity. Examining Figure 2(b), the porosity for subsets thicker than $60-80 \mu \mathrm{m}$ lie at the beginning of the plateau, showing that the selected subdomains were indeed not unduly influenced by the discrete GDL microstructure. Subsamples with thicknesses larger than $120 \mu \mathrm{m}$ were not analyzed due to their inability to meet the uniformity criterion of Eq. (5) over the high saturation gradients (and porosity variations) present across the full GDL [15]. As shown in Figure $3(\mathrm{c})$, the local tortuosity of the dry GDL, $\tau_{\text {loc }}^{\text {dry }}=\varepsilon_{\text {loc }} / f\left(\varepsilon_{\text {loc }}\right)$, computed for the selected subdomains $\left(L_{\mathrm{z}}=60-120 \mu \mathrm{m}\right)$ displayed a similar and consistent variation with porosity, regardless of their size. In contrast, preliminary tests conducted on 
subdomains with a thickness $L_{\mathrm{z}}=40 \mu \mathrm{m}$ deviated significantly from the results found for the thicker ones. The unrepresentative results computed on these overly small subsamples were discarded from subsequent analysis, and provided a reference lower bound for the selection of the aforementioned representative subdomains. 


\section{Discussion of Results}

\subsection{Local Dry Effective Diffusivity}

Figure 4 shows the numerically computed local effective diffusivity under dry conditions in both the through- and in-plane directions. The left panel shows the normalized dry effective diffusivity, $f_{\text {loc }}=D_{\text {loc }}^{\text {eff,dry }} / D_{\text {bulk }}$, as a function of local porosity, $\varepsilon_{\text {loc }}$, while the right panel shows the distribution of the exponent, $\mathrm{n}^{\mathrm{dry}}=\log \left(f_{\text {loc }}\right) / \log \left(\varepsilon_{\text {loc }}\right)$, obtained by fitting a power law to each individual data point $\left(\varepsilon_{\text {loc }}, f_{\text {loc }}\right)$. The computed results are marked by hollow symbols according to the thickness of the GDL subdomains; distinct colors are assigned to different porosity ranges.

As found in previous studies $[4,16,21,22,65-68]$, the GDL dry effective diffusivity is significantly lower than predicted by traditional models used in the literature for fibrous materials, namely the isotropic Bruggeman's effective medium theory [69], $f_{\text {loc }}=\varepsilon_{\text {loc }}^{1.5}$ and the anisotropic random fiber model of Tomadakis \& Sotirchos [20], $f_{\text {loc }}=$ $\varepsilon_{\text {loc }}\left[\frac{\varepsilon_{\text {loc }}-0.11}{1-0.11}\right]^{\mathrm{n}}$, with $\mathrm{n}$ equal to 0.785 and 0.521 for the through- and in-plane directions; both correlations are shown in Figure 4 by dashed and dot-dashed lines, respectively. The discrepancies of those models $[20,69]$ are explained by the oversimplified geometries used in their formulation as compared to the complex structure formed by fibers, binder and PTFE in carbon-paper GDLs $[16,21]$. The computed results are in agreement with the experimental data reported by Flückiger et al. [16] for Toray ${ }^{\circledR}$ TGP-H060 GDLs (black solid markers), which are morphologically comparable to the thicker Toray ${ }^{\circledR}$ TGP-H-120 used here to screen the subdomains (190 vs. $370 \mu \mathrm{m}$ ). Interestingly, the dependence of the effective diffusivity on local porosity due to the heterogeneous variations between subdomains behaves similarly to the data of Flückiger et al. [16], who measured the effective diffusivity on full GDLs subjected to different compression ratios (i.e., average porosities). 
The anisotropic nature of carbon-paper GDLs is clearly reflected in the different distributions of the power-law exponent $\mathrm{n}^{\text {dry }}$ obtained for the through- and in-plane directions. The effective diffusivity over the porosity range observed in the simulations $\left(\varepsilon_{\text {loc }} \approx 0.5-0.8\right)$ is well described by power laws of the form $f_{\text {loc }}=\varepsilon_{\text {loc }}^{\mathrm{n}^{\text {dry }}}$, with $\mathrm{n}^{\text {dry }}$ equal to $3.5\left(R^{2}=0.93\right)$ and $2.5\left(R^{2}=0.9\right)$ for the through- and in-plane directions, respectively. The good agreement with previous experimental data [16] provides support for the computed results and the methodology used here. The scatter of the results around the best-fit curves (regardless of the porosity range) can be attributed to changes in the local gas tortuosity throughout the GDL $[14,16]$; similar variations are observed in the global data of Flückiger et al. [16] due to the effect of PTFE loading.

\subsection{Local Relative Effective Diffusivity}

The results for the relative effective diffusivity, $g_{\text {loc }}\left(s_{\text {loc }}\right)$, and power-law exponent, $\mathrm{n}^{\text {wet, }}$, are shown in Figure 5 using a representation similar to that of Figure 4. The stochastic variations between data points at equal saturation levels are expected, considering that in each subdomain water is arranged in a random fashion. The uniformity factor (Eq. (5)) used to screen subdomains ensures that no large saturation gradients are present in the GDL subdomains, but it does not consider whether water is confined to a few large slugs or dispersed as many small clusters $[17,27]$. As a result, the power-law exponent $\mathrm{n}^{\text {wet }}$ characterizing the blockage effect of water displays a wider distribution compared to the dry results.

As seen in Figure $5(\mathrm{~b})$, the individual exponents $\mathrm{n}_{\mathrm{TP}}^{\text {wet }}$ fitted to each data point $\left(s_{\text {loc }}, g_{\text {loc }}\right)$ for the through-plane direction vary from 1.25 to 3.75 , with more than half of the data concentrated between 1.75 and 2.25. The exponent fitted to the full dataset is $\mathrm{n}_{\mathrm{TP}}^{\text {wet }}=2.15$, with $\mathrm{R}^{2}=0.8$. The power-law exponent $\mathrm{n}_{\mathrm{IP}}^{\text {wet }}$ for the in-plane direction shown in Figure 5(d) varies from 1 to 3.75 , with more than half of the values ranging between 1.75 and 2.5. The best-fit exponent to the full dataset is $\mathrm{n}_{\mathrm{IP}}^{\text {wet }}=2.25\left(\mathrm{R}^{2}=\right.$ 
0.75). These average exponents should be considered as the most representative values to characterize the local diffusive resistance of water under pressure-controlled invasion conditions. Compared to previous works in the field, values as high as $\mathrm{n}_{\mathrm{TP}}^{\text {wet }}=5$ were reported by Gostick et al. [28] for the through-plane direction, but as has been discussed previously, such values are not suitable for use in macroscopic models since they were obtained under non-uniform saturation conditions. The seminal pore-network work of Nam \& Kaviany [17] provides perhaps the best comparison to this work since they did not use invasion-percolation concepts to place water in their network, but considered heuristic algorithms resulting in a uniform saturation distribution. Nonetheless, they found exponents in the range of $\mathrm{n}_{\mathrm{TP}}^{\text {wet }}=3-4$ [17]. These higher values can be explained by the over-restrictive all-or-nothing algorithm used by those authors to fill the pores by water on the otherwise highly idealized pore structures (stacked screens) assumed in their work. Such a scenario [17] differs substantially from the real, finger-like water configuration and fibrous GDL considered here [15]. Interestingly, Nam \& Kaviany [17] along with numerous other groups $[15,18,19]$, have found $\mathrm{n}_{\mathrm{IP}}^{\text {wet }} \approx 2$ for the in-plane direction even for situations with steep saturation profiles across the GDL, in agreement with the present results.

The nearly isotropic character observed for the relative effective diffusivity, as both the though- and in-plane correlations follow an approximately quadratic power law, may be somewhat unexpected given the anisotropic pore structure of carbon-paper GDLs. This issue is examined in Figure 6 based on the average chord-length distributions of the dry and wet GDLs (see Figure 3(a) for details). Figure 6(a) shows the average chord length of the air space, $l_{\mathrm{a}, \mathrm{avg}}^{\mathrm{wet}}$, as a function of chord angle in the $x-z$ plane, $\theta$, for the complete range of applied capillary pressures, $p_{c}$; the inset represents the average chord length of water, $l_{\mathrm{w}, \mathrm{wavg}}^{\mathrm{wet}}$. As can be seen, the average size of the air space is reduced uniformly in all directions (i.e., for every angle $\theta$ ) as the capillary pressure (saturation level) in the GDL is increased. In other words, the curves shift downwards for a given $\theta$ in proportion to the size of the pore space available in that direction. This result can also be observed in the 
variation of the water chord length. It is seen that the size of water clusters is higher for the in-plane direction due to the larger size of pores in that direction. However, throat sizes in the GDL seem to be isotropic, so that water invades the pore space (i.e., the curves shift upwards) without a predominant direction. Note that above the breakthrough point $\left(p_{\mathrm{c}} \approx 3 \mathrm{kPa}\right)$, the average size of water clusters remains almost unaltered, due to the smaller radius of the pores invaded at this stage of the invasion process. As a result, the relative variation of the pore size between the wet and dry GDLs, $l_{\mathrm{a}, \mathrm{avg}}^{\mathrm{wet}} / l_{\mathrm{a}, \mathrm{avg}}^{\mathrm{dry}}$, shown in Figure $6(\mathrm{~b})$ is isotropic, that is, the relative reduction of the pore space is almost the same in any one direction at a given capillary pressure level. This analysis demonstrates that the (nearly) isotropic blockage effect of water observed under uniform saturation conditions is in fact consistent with the configuration of water clusters in the invasion experiments.

\subsection{Validation of Approach}

The effective diffusivity correlations proposed above are suitable to be used in macroscopic continuum models since they represent the impact of local porosity and saturation. It is prudent to confirm now if these correlations are able to reproduce the full-scale effective diffusivity data of GDLs with inhomogeneous porosity and saturation distributions. To this end, 1D global averaging rules are presented in Section 3.3.1 to estimate the global or equivalent effective diffusivity of full GDLs based on the porosity and saturation profiles across the material. In Section 3.3.2, the upscaling algorithm is then applied to the GDL sample from which the present subdomains were taken [15]. Finally, the generality of the proposed local correlations to recover the global properties reported in previous studies $[18,19]$ is tested in Section 3.3.3.

\subsubsection{Upscaling Algorithm}

In the invasion experiments, water was injected into one face and percolated in the 
through-plane direction, leading to strong saturation gradients across the GDL. Moreover, notable variations in porosity are present across the thickness of carbon-paper GDLs [15]. Therefore, introducing an electrical analogy, through-plane diffusion behaves as resistors in series, since gas must diffuse through each layer of the porosity/saturation profiles, whereas in-plane diffusion behaves as resistors in parallel, due to the perpendicular arrangement of water percolation and diffusive processes. This concept is illustrated in Figure 7(a). After subdividing the total GDL domain of size $L_{\mathrm{x}}^{\mathrm{T}} \times L_{\mathrm{y}}^{\mathrm{T}} \times L_{\mathrm{z}}^{\mathrm{T}}$ into $\mathrm{N}$ evenly distributed blocks of thickness $\Delta z$, the electrical circuit analogy $\left(R^{\text {eff }} \sim L / D^{\text {eff }} A\right)$ leads to:

$$
\begin{aligned}
& \text { TP: } R_{\mathrm{glb}, \mathrm{TP}}^{\mathrm{eff}}=\sum_{\mathrm{i}=1}^{\mathrm{N}} R_{\mathrm{loc}, \mathrm{TP}, \mathrm{i}}^{\mathrm{eff}} \Rightarrow \frac{L_{\mathrm{z}}^{\mathrm{T}}}{D_{\mathrm{glb}, \mathrm{TP}}^{\mathrm{eff}} L_{\mathrm{x}}^{\mathrm{T}} L_{\mathrm{y}}^{\mathrm{T}}}=\sum_{\mathrm{i}=1}^{\mathrm{N}} \frac{\Delta z}{D_{\mathrm{loc}, \mathrm{TP}, \mathrm{i}}^{\mathrm{eff}} L_{\mathrm{x}}^{\mathrm{T}} L_{\mathrm{y}}^{\mathrm{T}}} \\
& \mathrm{IP}: \frac{1}{R_{\mathrm{glb}, \mathrm{IP}}^{\mathrm{eff}}}=\sum_{\mathrm{i}=1}^{\mathrm{N}} \frac{1}{R_{\mathrm{loc}, \mathrm{IP}, \mathrm{i}}^{\mathrm{eff}}} \Rightarrow \frac{D_{\mathrm{glb}, \mathrm{IP}}^{\mathrm{eff}} L_{\mathrm{y}}^{\mathrm{T}} L_{\mathrm{z}}^{\mathrm{T}}}{L_{\mathrm{x}}^{\mathrm{T}}}=\sum_{\mathrm{i}=1}^{\mathrm{N}} \frac{D_{\mathrm{loc}, \mathrm{IP}, \mathrm{i}}^{\mathrm{eff}} L_{\mathrm{y}}^{\mathrm{T}} \Delta z}{L_{\mathrm{x}}^{\mathrm{T}}}
\end{aligned}
$$

where $R_{\mathrm{glb}}^{\mathrm{eff}}$ and $D_{\mathrm{glb}}^{\mathrm{eff}}$ are the global diffusive resistance and global effective diffusivity of the full GDL, and $R_{\text {loc,i }}^{\text {eff }}$ and $D_{\text {loc,i }}^{\text {eff }}$ are the local diffusive resistance and local effective diffusivity of each block, respectively. Writing Eq. (6) in terms of effective diffusivity and canceling out the lengths in the material plane, $L_{\mathrm{x}}^{\mathrm{T}}$ and $L_{\mathrm{y}}^{\mathrm{T}}$, results in:

$$
\begin{array}{ll}
\text { TP: } & D_{\text {glb,TP }}^{\text {eff }}=\left[\sum_{i=1}^{N} \frac{\left(\Delta z / L_{z}^{\mathrm{T}}\right)}{D_{\text {loc }, \mathrm{TP}, \mathrm{i}}^{\text {eff }}}\right]^{-1} \\
\text { IP: } & \mathrm{D}_{\text {glb,IP }}^{\text {eff }}=\sum_{\mathrm{i}=1}^{\mathrm{N}}\left(\Delta z / L_{\mathrm{z}}^{\mathrm{T}}\right) \mathrm{D}_{\text {loc }, \mathrm{IP}, \mathrm{i}}^{\text {eff }}
\end{array}
$$

Thus, the through-plane global effective diffusivity, $D_{\text {glb,TP }}^{\text {eff }}$, is equal to the harmonic average of $\mathrm{N} D_{\text {loc,TP }}^{\text {eff }}$ values, while the global in-plane effective diffusivity, $D_{\text {glb,IP }}^{\text {eff }}$, is equal to the arithmetic average of $\mathrm{N} D_{\text {loc,IP }}^{\text {eff }}$ values.

The above discrete upscaling rules can be taken to the continuum limit by considering that the GDL is composed of infinitely many differential resistors $(N \rightarrow \infty)$ of thickness $\mathrm{d} z^{*}[26]$ : 


$$
\begin{aligned}
& \text { TP: } \frac{D_{\text {glb,TP }}^{\text {eff }}}{D_{\text {bulk }}}=\left[\int_{0}^{1} f_{\text {loc }}^{-1}\left(\bar{\varepsilon}_{\mathrm{z}}\right) g_{\text {loc }}^{-1}\left(\bar{s}_{\mathrm{z}}\right) \mathrm{d} z^{*}\right]^{-1} \\
& \text { IP: } \frac{D_{\text {glb,IP }}^{\text {eff }}}{D_{\text {bulk }}}=\int_{0}^{1} f_{\text {loc }}\left(\bar{\varepsilon}_{\mathrm{z}}\right) g_{\text {loc }}\left(\bar{s}_{\mathrm{z}}\right) \mathrm{d} z^{*}
\end{aligned}
$$

where $z^{*}=z / L_{\mathrm{z}}^{\mathrm{T}}$ is the dimensionless $z$-coordinate. In this way, the global effective diffusivity, $D_{\text {glb }}^{\text {eff }} / D_{\text {bulk }}$, is unequivocally determined from the distribution of the local effective diffusivity, $D_{\text {loc }}^{\text {eff }}\left(z^{*}\right) / D_{\text {bulk }}=f_{\text {loc }}\left(\bar{\varepsilon}_{\mathrm{z}}\right) g_{\text {loc }}\left(\bar{s}_{\mathrm{z}}\right)$, corresponding to the specific porosity, $\bar{\varepsilon}_{\mathrm{Z}}\left(z^{*}\right)$, and saturation, $\bar{s}_{\mathrm{Z}}\left(z^{*}\right)$, profiles across the GDL. The harmonic averaging, Eq. (8), is analogous to solving a non-linear diffusion equation with variable diffusivity in the through-plane direction, while the arithmetic averaging, Eq. (9), is equivalent to considering linear diffusion at every (nearly homogeneous) $x-y$ plane perpendicular to the through-plane direction.

The upscaling algorithm represents the superimposition of two independent effects: (i) the macroscopic variations of porosity, $\bar{\varepsilon}_{\mathrm{z}}$, and saturation, $\bar{s}_{\mathrm{z}}$, through the GDL, and (ii) the relationships assumed for $f_{\text {loc }}\left(\bar{\varepsilon}_{\mathrm{z}}\right)$ and $g_{\text {loc }}\left(\bar{s}_{\mathrm{z}}\right)$, which depend on the local intrinsic diffusive properties of the porous medium. Macroscopic continuum models already predict/incorporate their own saturation/porosity distributions (effect i), so they require estimates of the local or intrinsic GDL effective diffusivity (effect ii). In the following sections, different options for $g_{\text {loc }}$ in Eq. (8) and Eq. (9) are compared to globally measured values for the relative effective diffusivity, $g_{\mathrm{glb}}$, as function of average (or total) saturation, $s_{\text {avg. }}$ In all the analysis, the functional relationships for the local dry effective diffusivity, $f_{\text {loc }}$, are kept equal to those determined in Section 3.1: $f_{\text {loc,TP }}=\bar{\varepsilon}_{\mathrm{z}}^{3.5}, f_{\text {loc,IP }}=$ $\bar{\varepsilon}_{\mathrm{Z}}^{2.5}$.

\subsubsection{Comparison with Original Tomographic Data}

Figure 7 (b)-(c) shows the global relative effective diffusivity, $g_{\mathrm{glb}}=D_{\mathrm{glb}}^{\text {eff,wet }} / D_{\mathrm{glb}}^{\text {eff,dry }}$, as a 
function of average saturation, $s_{\mathrm{avg}}$, in (a) the through-plane, and (b) the in-plane direction calculated with the above upscaling rules, along with the layer-scale effective properties computed previously for GDL domains with moderate peak saturation distributions [15]. Two different power laws are considered in the upscaling for the through-plane component $g_{\text {loc,TP }}\left(\bar{S}_{\mathrm{Z}}\right)$ : the nearly quadratic relationship, $g_{\text {loc,TP }}=$ $\left(1-\bar{s}_{\mathrm{Z}}\right)^{2.15}$, derived in Section 3.2, and the approximately cubic relationship, $g_{\text {loc,TP }}=$ $\left(1-\bar{s}_{\mathrm{Z}}\right)^{3.1}$, determined from the global data of the inhomogeneous full GDL [15]. Similarly, two correlations are considered for the in-plane direction to test their influence on the upscaling: the local correlation, $g_{\text {loc,IP }}=\left(1-\bar{s}_{\mathrm{Z}}\right)^{2.25}$, computed in Section 3.2, and a cubic power law, $g_{\text {loc,IP }}=\left(1-\bar{s}_{\mathrm{z}}\right)^{3}$.

Examining the through-plane results, good agreement is found between the global [15] and the upscaled properties when using the local correlation computed here. The upscaling of the saturation exponent from $\mathrm{n}_{\mathrm{TP}}^{\text {wet }}=2.15$ at the local scale to $\mathrm{n}_{\mathrm{TP}}^{\text {wet }}=3.1$ at the global scale is caused by the inhomogeneous saturation distribution across the $\mathrm{GDL}$, together with the nonlinear dependence of diffusive transport on local saturation. In contrast, when the cubic correlation obtained at the global scale [15] is directly used to describe local transport, the upscaled or equivalent GDL diffusivity is dramatically underestimated, leading to a global correlation $g_{\mathrm{glb}, \mathrm{TP}}=\left(1-s_{\mathrm{avg}}\right)^{5}$. Such a low global diffusivity is explained by the disproportionately high bottleneck caused by highsaturation regions in the upscaling, since the blockage effect of non-uniform saturation is evaluated twice: once in the assumed expression for the local effective diffusivity, and again in the upscaling algorithm, that is, when solving for diffusive transport as in macroscopic models. For the in-plane direction, the influence of the local relationship on the upscaled properties is significantly lower due to the perpendicular arrangement of the saturation gradients [15]. A nearly quadratic power law $\left(\mathrm{n}_{\mathrm{IP}}^{\text {wet }}=2.25\right)$ leads to a good characterization of local in-plane diffusion, which is approximately the same correlation at the global scale of the GDL $\left(\mathrm{n}_{\mathrm{IP}}^{\text {wet }}=2.15\right)$. 
The agreement observed between the upscaled and global properties provides strong support to the local correlations extracted from the GDL subdomains. The above results clearly demonstrate the need to describe local diffusive transport in macroscopic models using functional relationships determined under uniform saturation conditions.

\subsubsection{Comparison with Other Sources}

Focusing on the global properties reported by other authors, Figure 8 compares the results of the upscaling procedure against the global data computed by Gostick [18] from water drainage in pore-network simulations, and the data obtained by Rosén et al. [19] from CFD simulations on water distributions of operational fuel cells. The porosity and saturation profiles needed for the upscaling analysis were provided by the author in the first case [18], while they were taken from the supplementary material presented by the authors in the second [19].

As seen in Figure 8, only small deviations are found between the upscaled and global $[18,19]$ properties for the in-plane direction. A nearly quadratic power law $\left(\mathrm{n}_{\mathrm{IP}}^{\mathrm{wet}} \approx 2\right)$ gives a good representation of local in-plane diffusion, in agreement with the analysis performed on our full-scale tomography data [15]. In contrast, a wider range is observed for the through-plane direction. The power-law exponent required to fit the global data of Gostick [18] is $\mathrm{n}_{\mathrm{TP}}^{\text {wet }}=1.6$, which is in reasonable agreement with that computed here $\left(\mathrm{n}_{\mathrm{TP}}^{\text {wet }}=2.15\right)$, especially considering the idealized geometry used by Gostick [18]. When using $\mathrm{n}_{\mathrm{TP}}^{\text {wet }}=3$ at the local scale, the global diffusivity is severely underestimated, leading to a power law $g_{\mathrm{glb}, \mathrm{TP}}=\left(1-s_{\mathrm{avg}}\right)^{14}$. Note that such low upscaled diffusivities arise from the high saturation gradients computed by Gostick [18], so that an underestimation of the local diffusivity dramatically penalizes the global properties of the entire GDL. This result further demonstrates that a nearly quadratic power law $g_{\text {loc,TP }} \approx\left(1-s_{\text {loc }}\right)^{2}$ provides a good description of the blockage effect of water under capillary-driven conditions. 
Turning attention now to the through-plane data of Rosén et al. [19], a good match is found for the under-the-channel region by using the present power-law correlation $\left(\mathrm{n}_{\mathrm{TP}}^{\text {wet }}=2.15\right)$. Such agreement is consistent with the shapes of the saturation distributions reported by Rosén et al. [19] for the under-the-channel region, which closely resemble those observed in the invasion experiments [15]. However, the global under-the-rib properties are better captured with an exponent $n_{\mathrm{TP}}^{\text {wet }}$ between 3 and 4 . The higher local relative resistance under the rib is likely due to the more complex water configurations that originate from the coupled interactions between capillary transport, water condensation, and interference effects with the ribs [70-72]. For instance, the differences found between the channel and rib regions seem to be consistent with the recent studies of Iranzo et al. [24] and Owejan et al. [25]. Using a similar philosophy to the present upscaling procedure, those works reported correlations for the local effective diffusivity by combining the predictions of a macroscopic model with current density and neutron-imaging saturation measurements. Iranzo et al. [24] found $\mathrm{n}_{\mathrm{TP}}^{\text {wet }}=1.85$ considering saturation distributions from a fuel cell specially designed to minimize the effect of ribs in their calculations. In contrast, Owejan et al. [25] computed a much higher exponent $n_{\mathrm{TP}}^{\text {wet }}=3.6-4$ using a conventional fuel cell in which saturation was almost entirely concentrated under the rib. 


\section{Conclusions}

Previous work has demonstrated that the spatial distribution of water within the GDL, and not just the average amount, has a major impact on the effective gas diffusivity. The present work explored the repercussions of this finding on the widely used volumeaveraged continuum models of GDLs by specifically examining which type of data is suitable for use. Conceptually speaking, macroscopic continuum models need effective properties obtained under uniform saturation conditions to be consistent with the fact that each computational node in the GDL (where the effective properties are locally applied) has a single locally-uniform saturation value assigned to it. These conditions are almost impossible to satisfy experimentally since all water distributions contain some trace of the water injection or withdrawal history. As an alternative means of obtaining the necessary data, a methodology has been developed for extracting constitutive relationships for the local effective gas diffusivity from globally inhomogeneous materials. To this end, the lattice Boltzmann method was combined with X-ray computed tomography (XCT) images of dry and partially-saturated carbon-paper GDLs acquired during water-invasion experiments. A massive computational campaign was performed on physically representative GDL subdomains, which were specifically chosen to have nearly uniform porosity and saturation distributions.

The approach was first examined on dry materials and it was found that the variation of the dry effective diffusivity, $f_{\text {loc }}=D_{\text {loc }}^{\text {eff,dry }} / D_{\text {bulk, }}$, with local porosity, $\varepsilon_{\text {loc, }}$, agreed well with existing experimental data for GDLs of similar morphology. A correlation was determined as a power law of the form $f_{\text {loc }}=\varepsilon_{\text {loc }}^{\mathrm{n}^{\text {dry }}}$, with $\mathrm{n}_{\mathrm{TP}}^{\text {dry }}=3.5$ and $\mathrm{n}_{\mathrm{IP}}^{\text {dry }}=2.5$ for the through- and in-plane directions, respectively. Attention was then directed towards partially-saturated GDLs. The local relative effective diffusivity, $g_{\text {loc }}=D_{\text {loc }}^{\text {eff,wet }} / D_{\text {loc }}^{\text {eff,dry }}$, was found to be rather isotropic, following a nearly quadratic dependence on local saturation: $g_{\text {loc }}=\left(1-s_{\text {loc }}\right)^{\mathrm{n}^{\text {wet }}}$, with $\mathrm{n}_{\mathrm{TP}}^{\text {wet }}=2.15$ and $\mathrm{n}_{\mathrm{IP}}^{\text {wet }}=2.25$. 
As a consistency check, simple 1D upscaling rules were used to demonstrate that the local correlations were able to recover the global properties [15] obtained on the full GDL from which the present subdomains were extracted. The good agreement between the upscaled and global results confirmed the validity of the developed methodology and resulting correlations. To conclude the analysis, the same upscaling technique was used to explore the general applicability of the proposed correlations against two independent datasets taken from the literature. These datasets included pore-network results from a capillary-driven simulation, and CFD results on XCT images of water distributions in an operational fuel cell. Good agreement was found in the former case, as well in the latter case for the region under the channel. Local through-plane diffusive transport under the rib was more constricted, however, requiring power-law relationships of third- to fourth-order, $g_{\text {loc,TP }}=\left(1-s_{\text {loc }}\right)^{3 \cdots 4}$. This result suggests that the functional relationships computed here are suitable for the common case of capillary invasion, but are not necessarily general for all scenarios. This gap could be addressed by applying the present technique to additional tomographic images obtained on a wider variety of water configurations, such as condensed water and those resulting from interference effects with the rib walls. The impact of GDL compression due to the cell assembly process is also worth exploring.

An important issue that warrants closer attention is the physical validity of liquid water distributions predicted by macroscopic models to which the present correlations are meant to apply. The quantitative and qualitative discrepancies between such models (see, e.g, Figure 1(b)) and the real water profiles observed in neutron or X-ray imaging studies (see, e.g, [15]) are quite substantial. Numerous explanations can be invoked to understand the differences, but at least part of the discrepancies are due to the fact that capillary pressure curves used in such models are measured globally, in direct contradiction with the concepts discussed in this work. Additional efforts should be devoted to improve the two-phase predictive capabilities of macroscopic continuum models. 


\section{Acknowledgements}

The authors thank Dr. Jin Hyun Nam (Daegu University, South Korea), Dr. Massoud Kaviany (University of Michigan, USA) and Dr. Felix N. Büchi (Paul Scherrer Institut, Switzerland) for insightful discussions and comments concerning their works. The authors also thank Dr. Dula Parkinson and Dr. Alastair MacDowell at the Advanced Light Source (ALS) for help in obtaining the tomographic images, as well as the support team of Calcul Québec and Compute Canada for their help during the simulation campaign. This work was supported by the Natural Science and Engineering Research Council of Canada's Discovery Grant program, the Assistant Secretary for Energy Efficiency and Renewable Energy, Fuel Cell Technologies Office, of the U. S. Department of Energy under contract DE-AC02-05CH11231, and Project ENE2011-24574 of the Spanish Ministerio de Economía y Competitividad (MEC). XCT experiments were performed on beamline 8.3.2 at the ALS, Lawrence Berkeley National Laboratory, which is a national user facility funded by the Department of Energy, Office of Basic Energy Sciences under contract DE-AC02-05CH11231. Numerical calculations were conducted on the supercomputing clusters Guillimin and Colosse managed by Calcul Québec and Compute Canada. The operation of these supercomputers is funded by the Canada Foundation for Innovation (CFI), Ministère de l'Économie, de l'Innovation et des Exportations du Québec (MEIE), RMGA and the Fonds de recherche du Québec - Nature et technologies (FRQ-NT). 


\section{Nomenclature}

\section{Symbols}

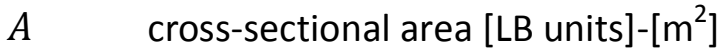

C mass concentration [LB units]-[mol $\left./ \mathrm{m}^{3}\right]$

$D \quad$ mass diffusivity [LB units]-[ $\mathrm{m}^{2} / \mathrm{s}$ ]

$D_{\text {c }} \quad$ capillary diffusivity $[\mathrm{kg} / \mathrm{m} \mathrm{s}]$; see Figure 1

F $\quad$ Faraday's constant $[\mathrm{C} / \mathrm{mol}]$; see Figure 1

$f(\varepsilon) \quad$ (normalized) dry effective diffusivity function; see Eq. (2)

$g(s) \quad$ (normalized) relative effective diffusivity function; see Eq. (2)

I current density $\left[\mathrm{A} / \mathrm{m}^{2}\right]$; see Figure 1

$J(s) \quad$ Leverett J-function; see Figure 1

$j \quad$ Fickian diffusion flux [LB units]-[mol$\left./ \mathrm{m}^{2} \mathrm{~s}\right]$

$K \quad$ absolute permeability $\left[\mathrm{m}^{2}\right]$; see Figure 1

$k_{\mathrm{rl}} \quad$ liquid-phase relative permeability; see Figure 1

$L_{\mathrm{i}} \quad$ length in i-direction [LB units]-[m]

$L_{\mathrm{i}}^{\mathrm{T}} \quad$ total length in i-direction [m]

$l_{\mathrm{i}} \quad$ chord length of phase $\mathrm{i}[\mathrm{m}]$

$M \quad$ molecular weight $[\mathrm{kg} / \mathrm{mol}]$; see Figure 1

$\mathrm{N} \quad$ arbitrary number

n power-law exponent

$p \quad$ pressure $[\mathrm{Pa}]$

$p_{\mathrm{c}} \quad$ capillary pressure $[\mathrm{Pa}]$

$R \quad$ diffusive resistance $\left[\mathrm{s} / \mathrm{m}^{3}\right]$

$S \quad$ liquid (water) saturation

$\bar{S}_{\mathrm{z}}\left(z_{\mathrm{i}}\right) \quad x y$-averaged liquid saturation at position $z=z_{\mathrm{i}}$

UF uniformity factor; see Eq.(5)

$x \quad$ in-plane coordinate [LB units]-[m] 


$$
\begin{array}{ll}
y & \text { secondary in-plane coordinate [LB units]-[m] } \\
z & \text { through-plane coordinate [LB units]-[m] }
\end{array}
$$

\begin{tabular}{|c|c|}
\hline$\Delta$ & spatial difference or increment [m] \\
\hline$\delta_{\mathrm{z}}$ & GDL thickness window length [m]; see [15] \\
\hline$\varepsilon$ & (dry) porosity \\
\hline$\varepsilon_{\mathrm{v}}$ & void volume fraction; see Eq. (1) \\
\hline $\bar{\varepsilon}_{\mathrm{z}}\left(z_{\mathrm{i}}\right)$ & $x y$-averaged porosity at position $z=z_{\mathrm{i}}$ \\
\hline$\theta$ & direction angle $\left[{ }^{\circ}\right]$ \\
\hline$\theta_{\mathrm{c}}$ & contact angle $\left[{ }^{\circ}\right]$; see Figure 1 \\
\hline & mute variable in Eq. (5) \\
\hline & kinematic viscosity $\left[\mathrm{m}^{2} / \mathrm{s}\right]$; see Figure 1 \\
\hline & density $\left[\mathrm{kg} / \mathrm{m}^{3}\right]$; see Figure 1 \\
\hline & interfacial tension [N/m]; see Figure 1 \\
\hline & tortuosity; see Eq.(1) \\
\hline
\end{tabular}

\section{Greek letters}

\section{Subscripts}

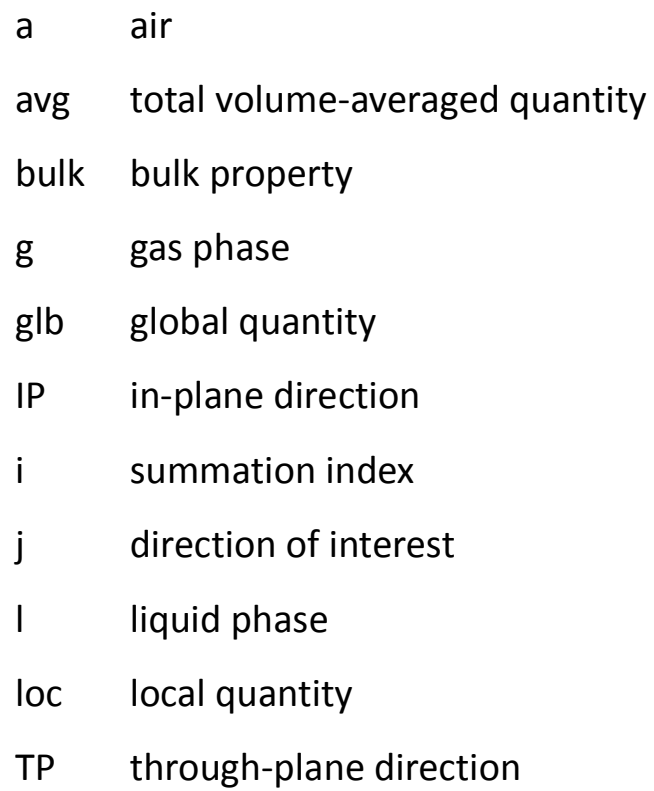


w water

\section{Superscripts}

eff effective

dry dry conditions

max maximum

wet wet or partially-saturated conditions

* dimensionless or normalized value 


\section{References}

[1] M.W. Ellis, M.R. Von Spakovsky, D.J. Nelson, Proc. IEEE. 89 (2001) 1808-1818.

[2] A.Z. Weber, J. Newman, J. Electrochem. Soc. 152 (2005) A677-A688.

[3] J.T. Gostick, M.A. Ioannidis, M.W. Fowler, M.D. Pritzker, Electrochem. Commun. 11 (2009) 576-579.

[4] G.S. Hwang, A.Z. Weber, J. Electrochem. Soc. 159 (2012) F683-F692.

[5] A.D. Santamaria, P.K. Das, J.C. MacDonald, A.Z. Weber, J. Electrochem. Soc. 161 (2014) F1184-F1193.

[6] A.Z. Weber, R.L. Borup, R.M. Darling, P.K. Das, T.J. Dursch, W. Gu, D. Harvey, A. Kusoglu, S. Litster, M.M. Mench, R. Mukundan, J.P. Owejan, J.G. Pharoah, M. Secanell, I.V. Zenyuk, J. Electrochem. Soc. 161 (2014) F1254-F1299.

[7] C.-Y. Wang, Chem. Rev. 104 (2004) 4727-4765.

[8] U. Pasaogullari, C.-Y. Wang, J. Electrochem. Soc. 151 (2004) A399-A406.

[9] P.A. García-Salaberri, M. Vera, R. Zaera, Int. J. Hydrogen Energy 36 (2011) 1185611870.

[10] P.A. García-Salaberri, M. Vera, I. Iglesias, J. Power Sources 246 (2014) 239-252.

[11] P.A. García-Salaberri, M. Vera, J. Power Sources 285 (2015) 543-558.

[12] N. Zamel, X. Li, Prog. Energy Combust. Sci. 39 (2013) 111-146.

[13] R. Koresawa, Y. Utaka, Int. J. Heat Mass Transf. 76 (2014) 549-558.

[14] M.M. Mezedur, M. Kaviany, W. Moore, AIChE J. 48 (2002) 15-24.

[15] P.A. García-Salaberri, G. Hwang, M. Vera, A.Z. Weber, J.T. Gostick, Int. J. Heat Mass Transf. 86 (2015) 319-333.

[16] R. Flückiger, S.A. Freunberger, D. Kramer, A. Wokaun, G.G. Scherer, F.N. Büchi, Electrochim. Acta 54 (2008) 551-559.

[17] J.H. Nam, M. Kaviany, Int. J. Heat Mass Transf. 46 (2003) 4595-4611. 
[18] J.T. Gostick, J. Electrochem. Soc. 160 (2013) F731-F743.

[19] T. Rosén, J. Eller, J. Kang, N.I. Prasianakis, J. Mantzaras, F.N. Büchi, J. Electrochem. Soc. 159 (2012) F536-F544.

[20] M.M. Tomadakis, S.V. Sotirchos, AIChE J. 39 (1993) 397-412.

[21] T.D. Myles, A.A. Peracchio, U. Pasaogullari, W.K.S. Chiu, J. Electrochem. Soc. 162 (2015) F645-F650.

[22] N. Zamel, X. Li, J. Shen, Energy \& Fuels. 23 (2009) 6070-6078.

[23] I.V. Zenyuk, D.Y. Parkinson, G. Hwang, A.Z. Weber, Electrochem. Commun. 53 (2015) 24-28.

[24] A. Iranzo, P. Boillat, P. Oberholzer, J. Guerra, Energy 68 (2014) 971-981.

[25] J.P. Owejan, T.A. Trabold, M.M. Mench, Int. J. Heat Mass Transf. 71 (2014) 585592.

[26] J. Bear, J.M. Buchlin, Modeling and Application of Transport Phenomena in Porous Media, Kluwer Academic Publishers, Boston (1991).

[27] N.S. Martys, Mater. Struct. 32 (1999) 555-562.

[28] J.T. Gostick, M.A. Ioannidis, M.W. Fowler, M.D. Pritzker, J. Power Sources 173 (2007) 277-290.

[29] N. Zamel, X. Li, J. Becker, A. Wiegmann, Int. J. Hydrogen Energy 36 (2011) 54665478.

[30] J. Becker, V. Schulz, A. Wiegmann, J. Fuel Cell Sci. Technol. 5 (2008) 021006 1-9.

[31] I.S. Hussaini, C.Y. Wang, J. Power Sources 195 (2010) 3830-3840.

[32] X. Wang, T. Van Nguyen, D.S. Hussey, D.L. Jacobson, J. Electrochem. Soc. 157 (2010) B1777-B1782.

[33] O. Burheim, P.J.S. Vie, J.G. Pharoah, S. Kjelstrup, J. Power Sources 195 (2010) 249-256.

[34] P. Rama, Y. Liu, R. Chen, H. Ostadi, K. Jiang, X. Zhang, R. Fisher, M. Jeschke, J. Fuel Cell Sci. Technol. 7 (2010) 031015. 
[35] P. Rama, Y. Liu, R. Chen, H. Ostadi, K. Jiang, X. Zhang, Y. Gao, P. Grassini, D. Brivio, Int. J. Numer. Meth. Fluids 67 (2011) 518-530.

[36] Y. Gao, X. Zhang, P. Rama, R. Chen, H. Ostadi, K. Jiang, J. Fuel Cell Sci. Technol. 9 (2012) 041010.

[37] Y. Gao, X.X. Zhang, P. Rama, Y. Liu, R. Chen, H. Ostadi, K. Jiang, Fuel Cells 12 (2012) 365-381.

[38] H. Ostadi, P. Rama, Y. Liu, R. Chen, X.X. Zhang, K. Jiang, Chem. Eng. Sci. 65 (2010) 2213-2217.

[39] J. Park, M. Matsubara, X. Li, J. Power Sources 173 (2007) 404-414.

[40] J. Park, X. Li, J. Power Sources 178 (2008) 248-257.

[41] A. Nabovati, E.W. Llewellin, A.C.M. Sousa, Composites Part A 40 (2009) 860-869.

[42] A. Nabovati, J. Hinebaugh, A. Bazylak, C.H. Amon, J. Power Sources 248 (2014) 83-90.

[43] S.H. Kim, H. Pitsch, J. Electrochem. Soc. 156 (2009) B673-B681.

[44] M. Van Doormaal, J. Pharoah, Int. J. Numer. Meth. Fluids 59 (2009) 75-89.

[45] L. Hao, P. Cheng, J. Power Sources 186 (2009) 104-114.

[46] J. Yablecki, A. Nabovati, A. Bazylak, J. Electrochem. Soc. 159 (2012) B647-B653.

[47] D. Froning, J. Brinkmann, U. Reimer, V. Schmidt, W. Lehnert, D. Stolten, Electrochim. Acta 110 (2013) 325-334.

[48] U.R. Salomov, E. Chiavazzo, P. Asinari, Comput. Math. with Appl. 67 (2014) 393411.

[49] X.-D. Niu, T. Munekata, S.-A. Hyodo, K. Suga, J. Power Sources 172 (2007) 542552.

[50] V.P. Schulz, J. Becker, A. Wiegmann, P.P. Mukherjee, C.-Y. Wang, J. Electrochem. Soc. 154 (2007) B419-B426.

[51] T. Koido, T. Furusawa, K. Moriyama, J. Power Sources 175 (2008) 127-136.

[52] L. Hao, P. Cheng, Int. J. Heat Mass Transf. 53 (2010) 1908-1913. 
[53] J. Yablecki, J. Hinebaugh, A. Bazylak, J. Electrochem. Soc. 159 (2012) F805-F809.

[54] S. Succi, The Lattice-Boltzmann Equation for Fluid Dynamics and Beyond, Oxford University Press, New York (2001).

[55] F. Higuera, J. Jimenez, Europhys. Lett. 9 (1989) 663-668.

[56] Palabos 1.3r0, FlowKit Ltd. (http://www.palabos.org/).

[57] P.L. Bhatnagar, E.P. Gross, M. Krook, Phys. Rev. 94 (1954) 511-525.

[58] Q. Zou, X. He, Phys. Fluids 9 (1997) 1591-1598.

[59] D.A. Wolf-Gladrow, Lattice Gas Cellular Automata and Lattice Boltzmann Models, Springer, Berlin (2000).

[60] http://calculquebec.ca

[61] F.A.L. Dullien, Porous Media: Fluid Transport and Pore Structure, Academic Press, Inc., San Diego (1992).

[62] Z. Fishman, J. Hinebaugh, A. Bazylak, J. Electrochem. Soc. 157 (2010) B1643B1650.

[63] J. Roth, J. Eller, F. Marone, F.N. Büchi, J. Phys. Chem. C 117 (2013) 25991-25999.

[64] S. Torquato, Random Heterogenous Materials: Microstructure and Macroscopic Properties, Springer, New York (2002).

[65] J.M. LaManna, S.G. Kandlikar, Int. J. Hydrogen Energy 36 (2011) 5021-5029.

[66] M. Möst, M. Rzepka, U. Stimming, J. Power Sources 191 (2009) 456-464.

[67] M.J. Martínez, S. Shimpalee, J.W. Van Zee, J. Electrochem. Soc. 156 (2009) B80B85.

[68] R. Rashapov, F. Imami, J.T. Gostick, Int. J. Heat Mass Transf. 85 (2015) 367-374.

[69] D.A.G. Bruggeman, Annalen der Physik 5 (1935) 636-664.

[70] J. Eller, T. Rosén, F. Marone, M. Stampanoni, A. Wokaun, F.N. Büchi, J. Electrochem. Soc. 158 (2011) B963-B970.

[71] J. Hinebaugh, A. Bazylak, J. Electrochem. Soc. 157 (2010) B1382-B1390. 


\section{Tables and Figures}

Figure 1: (a) The gray blocks labeled A, B \& C represent three experiments conducted on GDL samples with nearly uniform saturation ( $s_{\text {avg }} \approx s_{\text {unif }}$ ), while the gray blocks labeled D, E \& F represent three experiments conducted on samples with gradient saturation profiles. The dashed arrows indicate the direction of diffusion. The effective diffusivity, $D^{\text {eff }} / D_{\text {bulk, }}$, is shown as a function of average saturation, $s_{\text {avg }}$, for all six experiments on the right plot. (b) Distribution of local water saturation, $s_{\text {loc }}$, through the GDL (z-dir.) computed according to the homogenized unsaturated flow theory $[8,17,26]: \nabla \cdot\left(D_{c} \nabla s_{\text {loc }}\right)=$ 0 , with capillary diffusivity $D_{\mathrm{c}}=-\left(K k_{\mathrm{rl}} / v_{\mathrm{w}}\right)\left(\mathrm{d} p_{\mathrm{c}} / \mathrm{d} s_{\text {loc }}\right)$, and boundary conditions $s_{\text {loc }}(0)=0$ and $\left.D_{c} \nabla s_{\text {loc }}\right|_{z^{*}=1}=M_{\mathrm{w}} I / 2 F$. The location of the flow distributor and the catalyst layer is also indicated. Macroscopic models solving transport processes in the GDL require effective properties obtained under uniform saturation conditions (i.e., experiments A, B \& C, and not D, E \& F) to model every local homogenous node $\left(s_{\text {loc }}=s_{\text {avg }}^{\text {node }}\right.$ ) in which the GDL is divided (dotted box). Simulation parameters [8]: $L_{\mathrm{z}, \mathrm{GDL}}^{\mathrm{T}}=300 \mu \mathrm{m} ; I=1.4 \mathrm{~A} / \mathrm{cm}^{2} ; v_{\mathrm{w}}=3.65 \times 10^{-7} \mathrm{~m}^{2} / \mathrm{s}$ at $80^{\circ} \mathrm{C} ; K=6.875 \times 10^{-13} \mathrm{~m}^{2} ;$ $k_{\mathrm{rl}}=s_{\mathrm{loc}}{ }^{3} ; p_{\mathrm{c}}=p_{\mathrm{g}}-p_{\mathrm{l}}=\sigma \cos \theta_{c}(\varepsilon / K)^{1 / 2} J\left(s_{\mathrm{loc}}\right) \mathrm{Pa}$, with $\sigma=0.0625 \mathrm{~N} / \mathrm{m}, \theta_{c}=100^{\circ}, \varepsilon=0.5$, $J\left(s_{\text {loc }}\right)=1.417 s_{\text {loc }}-2.120 s_{\text {loc }}^{2}+1.263 s_{\text {loc }}^{3}$

Figure 2: (a) Schematic of the methodology used to compute functional relationships for the local dry, $f_{\text {loc }}$, and relative, $g_{\text {loc, }}$ effective diffusivities on local porosity, $\varepsilon_{\text {loc }}$, and local saturation, $s_{\text {loc, }}$, respectively. LB simulations were performed on XCT images of arbitrarily selected subdomains that are representative of the GDL microstructure and have nearly uniform porosity and saturation distributions. The rendered image stack corresponds to the breakthrough point $\left(p_{\mathrm{c}} \approx 3 \mathrm{kPa}\right)$. The direction of water injection in the invasion experiments is also indicated. (b) and (c) Representative elementary volume (REV) determinations of (b) porosity, and (c) water saturation at the breakthrough point for Toray ${ }^{\circledR}$ TGP$\mathrm{H}-120$ carbon paper. The $y$-axis shows the range of values calculated inside randomly drawn boxes of different thickness $L_{z}$ as indicated on the $x$-axis; the length in the material plane is kept equal to $L_{\mathrm{x}}=L_{\mathrm{y}}=400 \mu \mathrm{m}$. For the analysis of the porosity the outer high-porosity surface regions of the GDL were included, while for the water saturation only the core region was used. The unrepresentative microscopic scale (highly influenced by the granularity of the material) and the representative macroscopic scale (leading to consistent functional relationships for the effective diffusivity) are indicated in (b).

Figure 3: (a) Cross-sectional view of the pore space of the dry GDL, showing the air chord distribution for different direction angles, $\theta$, in the $x-z$ plane; $\theta=0^{\circ}$ corresponds to the through-plane (TP) direction, and $\boldsymbol{\theta}=90^{\circ}$ to the in-plane (IP) direction. For clarity in the presentation, the thickness and spacing of the white chords (labeled 1) are increased to 3 and 15 voxels, respectively. The solid phase of the GDL (fibers, binder and PTFE) is shown in brown, although in the calculations it is not distinguished from the background black air phase (labeled 0). To prevent artifacts due to the finite size of the images, chords intercepting the edges in the material plane are removed; however, chords intercepting the top and bottom faces in the TP direction are retained since these pores are naturally truncated at the 
sample surface. (b) Air chord-length distribution of the dry GDL, $l_{\mathrm{a}}^{\text {dry }}$, in the TP and IP directions computed on the $1.3 \times 1.3 \times 0.28 \mathrm{~mm}$ sample used to screen the GDL subdomains. The dots indicate the average chord lengths in both directions: $l_{\mathrm{a}, \mathrm{avg}, \mathrm{TP}}^{\mathrm{dry}}=31.24 \mu \mathrm{m}, l_{\mathrm{a}, \text { avg,IP }}^{\mathrm{dry}}=52.64 \mu \mathrm{m}$. Only subdomains that are representative of the GDL microstructure with TP lengths, $L_{\mathrm{z}}$, ranging from 60 to $120 \mu \mathrm{m}$ are considered for analysis. The extent in the material plane $\left(L_{x} \times L_{y}\right)$ is kept equal to $400 \times 400 \mu \mathrm{m}^{2}$. (c) Local gas tortuosity of the dry GDL, $\tau_{\text {loc }}^{\text {dry }}=\varepsilon_{\text {loc }} / f\left(\varepsilon_{\text {loc }}\right)$, in the TP (up) and IP (down) directions corresponding to the selected subdomains $\left(L_{\mathrm{z}}=60,80,93,106 \& 120 \mu \mathrm{m}\right)$, as compared to the discarded subdomains of thickness $L_{\mathrm{z}}=\mathbf{4 0} \mu \mathrm{m}$. The fit to a power law of the form $\tau_{\text {loc }}^{\text {dry }}=\varepsilon_{\text {loc }}^{\mathrm{n}}$ to both datasets is shown by solid and dashed lines, respectively.

Figure 4: Left panel: computed local dry effective diffusivity, $f_{\text {loc }}=D_{\text {loc }}^{\text {eff,dry }} / D_{\text {bulk, }}$, as a function of local average porosity, $\varepsilon_{\text {loc }}$, for (a) the through-plane (TP), and (b) the in-plane (IP) direction, and comparison with previous data presented in the literature: Bruggeman's effective medium theory [69] (black dashed line), random fiber model of Tomadakis \& Sotirchos [20] (black dot-dashed line), and the experiments of Flückiger et al. [16] for Toray ${ }^{\circledR}$ TGP-H-060 carbon paper with different PTFE contents (black solid symbols). The computed results are marked with hollow symbols according to the thickness of the GDL subdomains $\left(L_{\mathrm{z}}=60-120 \mu \mathrm{m}\right)$ in the LB simulations, including the best fit to a power law of the form $f_{\text {loc }}=\varepsilon_{\text {loc }}^{\text {dry }}$. Right panel: distribution of the power-law exponent, $\mathbf{n}^{\text {dry }}=\log \left(f_{\operatorname{loc}}\right) / \log \left(\varepsilon_{\text {loc }}\right)$, associated to each data point $\left(\varepsilon_{\text {loc }}, f_{\text {loc }}\right)$ presented on the left panel for (b) the TP, and (d) the IP direction. The TP and IP results are, respectively, colored in reddish and bluish tones according to their porosity range: $\varepsilon_{\text {loc }}=0.5-0.6, \varepsilon_{\text {loc }}=0.6-0.7$, and $\varepsilon_{\text {loc }}=0.7-0.8$.

Figure 5: Left panel: computed local relative effective diffusivity, $g_{\text {loc }}=D_{\text {loc }}^{\text {eff,wet }} / D_{\text {loc }}^{\text {effy }}$, for (a) the through-plane (TP), and (c) the in-plane (IP) direction, as a function of local average saturation, $s_{\text {loc }}$. The results are marked with hollow symbols according to the thickness of the subdomains $\left(L_{\mathrm{z}}=60-\right.$ $120 \mu \mathrm{m})$ in the LB simulations, including the best fit to a power law of the form $g_{\text {loc }}=\left(1-s_{\text {loc }}\right)^{\mathrm{n}^{\text {wet }}}$. For reference purposes, other power-law relationships of similar form are shown by dashed lines; the space between the curves delimiting the numerical results is colored in grey. Right panel: distribution of the power-law exponent, $n^{\text {wet }}=\log \left(g_{\text {loc }}\right) / \log \left(1-s_{\text {loc }}\right)$, associated to each data point $\left(s_{\text {loc }}, g_{\text {loc }}\right)$ presented on the left panel for (b) the TP, and (d) the IP direction. The TP and IP results are, respectively, colored in reddish and bluish tones according to their saturation range: $s_{\text {loc }}=0-0.3, s_{\text {loc }}=0.3-$ 0.6 , and $s_{\text {loc }}=0.6-1$.

Figure 6: (a) Average air chord length, $l_{\mathrm{a}, \mathrm{avg}}^{\mathrm{wet}}$, as a function of direction angle, $\theta$, at different applied capillary pressures, $\boldsymbol{p}_{\mathrm{c}}$. The average saturation level, $\boldsymbol{s}_{\text {avg }}$, at each capillary pressure is also indicated. The direction angle starts from the through-plane (TP) direction $\left(\theta=0^{\circ}\right)$ and expands a rotation of $180^{\circ}$ in the $x-z$ plane; $\theta=90^{\circ}$ corresponding to the in-plane (IP) direction (see schematic in Figure 3(a)). The inset shows the variation of the average water chord length, $l_{\mathrm{w}, \text { avg }}(\theta)$. (b) Relative variation of the average air chord length between the partially-saturated and dry GDLs, $l_{\mathrm{a}, \mathrm{avg}}^{\mathrm{wet}} / l_{\mathrm{a}, \mathrm{avg}}^{\mathrm{dry}}$, as a function of direction angle, $\theta$. The breakthrough point in the invasion experiments was reached at $\boldsymbol{p}_{\mathrm{c}} \approx 3 \mathrm{kPa}$. The chord length distributions were computed on the $1.3 \times 1.3 \times 0.28 \mathrm{~mm}$ GDL sample from which the present subdomains were taken. 
Figure 7: (a) Schematic diagram illustrating the basis of the 1D upscaling algorithm used to estimate the global effective diffusivity, $D_{\mathrm{glb}}^{\text {eff }}$, of the inhomogeneous full GDL from the local effective diffusivity, $D_{\text {loc }}^{\text {eff }}$, distribution through the porous medium. Left: porosity and water saturation distributions across the GDL (z-dir.) due to through-plane water injection, showing the geometrical parameters of the modeled system. Right: discrete resistor network for diffusion in the through- (TP) and in-plane (IP) directions after subdividing the full GDL of size $L_{\mathrm{x}}^{\mathrm{T}} \times L_{\mathrm{y}}^{\mathrm{T}} \times L_{\mathrm{z}}^{\mathrm{T}}$ into $\mathrm{N}$ evenly distributed blocks of thickness $\Delta z$. The global effective diffusivities in the TP and IP directions are obtained, respectively, by harmonic and arithmetic averaging at the continuum limit $\left(\mathrm{N} \rightarrow \infty\right.$ resistors of thickness $\left.\mathbf{d z}^{*}\right)$ the local effective diffusivity distribution, $D_{\text {loc }}^{\text {eff }}\left(z^{*}\right)$, associated to the porosity, $\bar{\varepsilon}_{\mathbf{z}}\left(\mathbf{z}^{*}\right)$, and saturation, $\bar{s}_{\mathrm{z}}\left(\mathbf{z}^{*}\right)$, profiles across the GDL; $z^{*}=z / L_{\mathrm{z}}^{\mathrm{T}}$ is the dimensionless $z$-coordinate. (b) and (c) Global relative effective diffusivity, $g_{\mathrm{glb}}=D_{\mathrm{glb}}^{\text {eff,wet }} / D_{\mathrm{glb}}^{\text {eff,dry }}$, in (b) the TP, and (c) the IP direction, as a function of average saturation, $s_{\text {avg }}$, calculated by the upscaling rules given in Eq. (8) and Eq. (9), and comparison with the global data presented in [15] for GDL window lengths, $\delta_{\mathrm{z}} / L_{\mathrm{z}}^{\mathrm{T}}$, between 47 and $75 \%$. Different correlations are examined in the upscaling for the local relative effective diffusivity, $g_{\text {loc }}\left(\overline{\boldsymbol{s}}_{\mathrm{z}}\right)$, while the functional relationships for the local dry effective diffusivity are kept equal to those presented in Figure 4: $f_{\text {loc,TP }}=\bar{\varepsilon}_{\mathrm{z}}^{3.5}, f_{\text {loc,IP }}=\bar{\varepsilon}_{\mathrm{z}}^{2.5}$. The comparison for other window lengths [15] is omitted for clarity although they lead to similar conclusions.

Figure 8: Global relative effective diffusivity, $g_{\mathrm{glb}}=D_{\mathrm{glb}}^{\text {eff,wet }} / D_{\mathrm{glb}}^{\text {eff,dry }}$, as a function of average saturation, $s_{\text {avg }}$, calculated by the upscaling rules in Eq. (8) and Eq. (9), as compared to the global results computed by Gostick [18] ((a)-(b)) from water-drainage pore-network simulations, and by Rosén et al. [19] ((c)-(d)) from pore-scale CFD simulations on tomographic images of the under-the-channel and under-the-rib regions of an operational fuel cell. The left and right panels show the results in the through- (TP), and in-plane (IP) directions, respectively. Different correlations are considered in the upscaling for the local relative effective diffusivity, $g_{\text {loc }}\left(\bar{s}_{\mathrm{z}}\right)$, while the functional relationships for the local dry effective diffusivity are kept equal to those presented in Figure 4: $f_{\text {loc,TP }}=\bar{\varepsilon}_{\mathrm{z}}^{3.5}, f_{\text {loc,IP }}=\bar{\varepsilon}_{\mathrm{z}}^{2.5}$. 


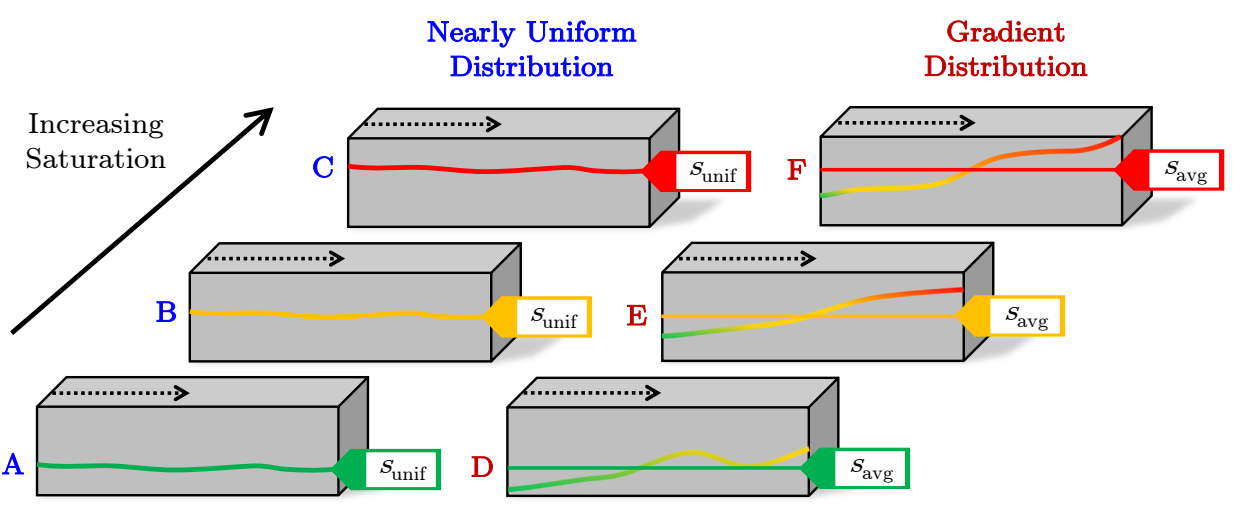

(b)

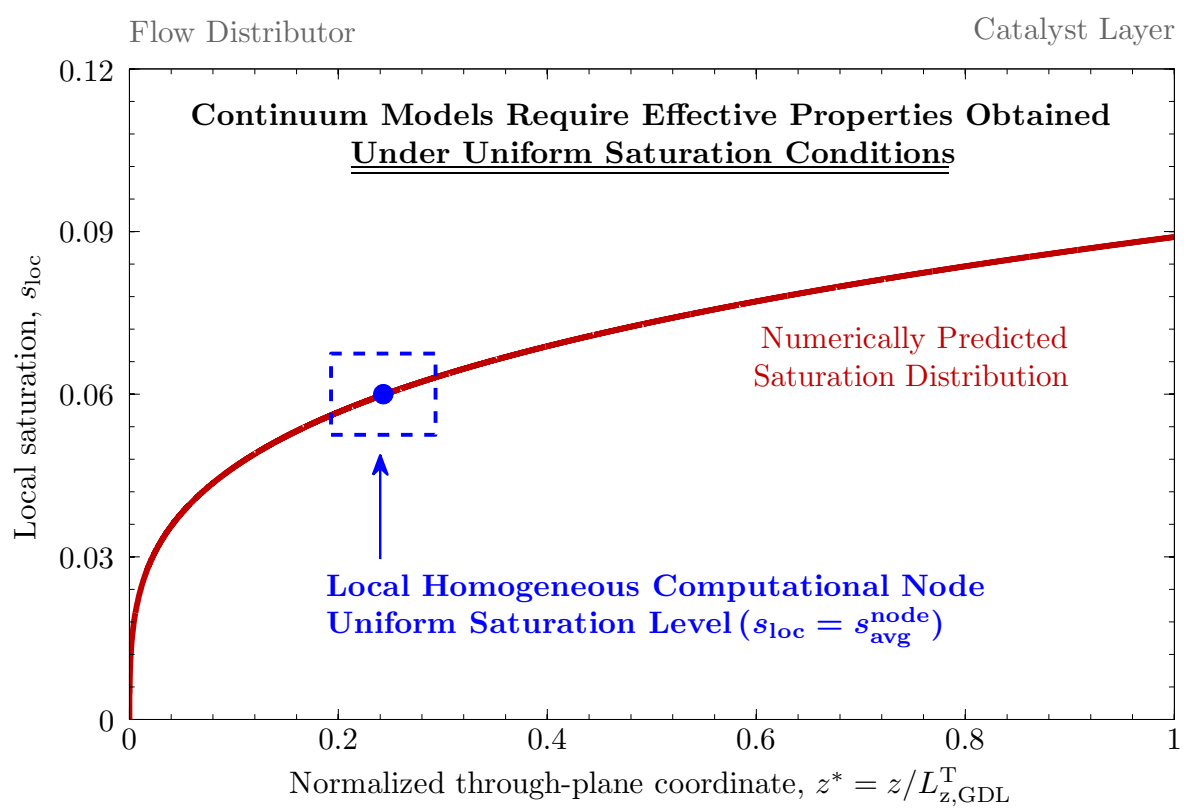


(a)

\begin{abstract}
Arbitrary Subdomains
Consider those that are representative $\&$ have

nearly uniform porosity/saturation distributions
\end{abstract}
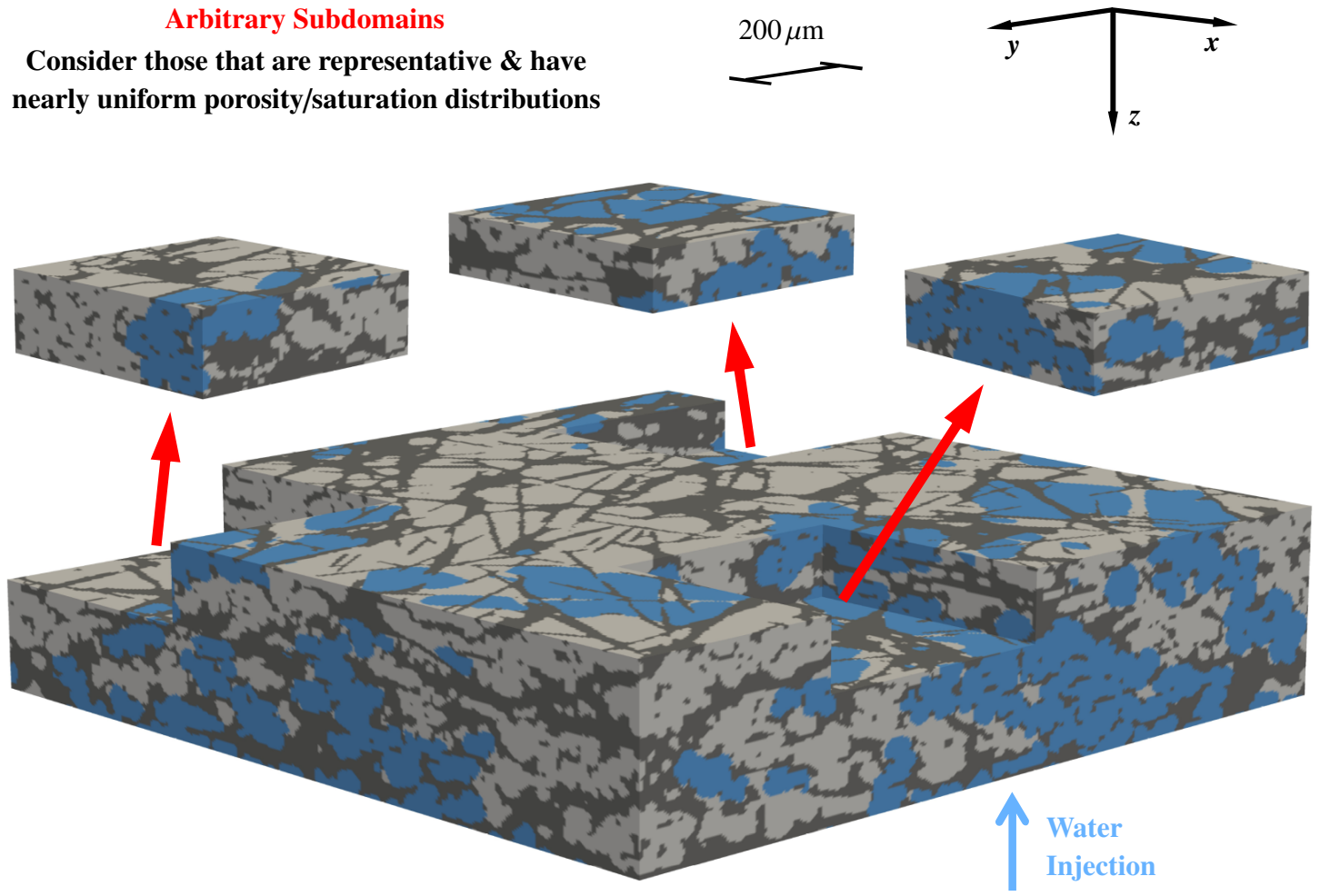

(b)

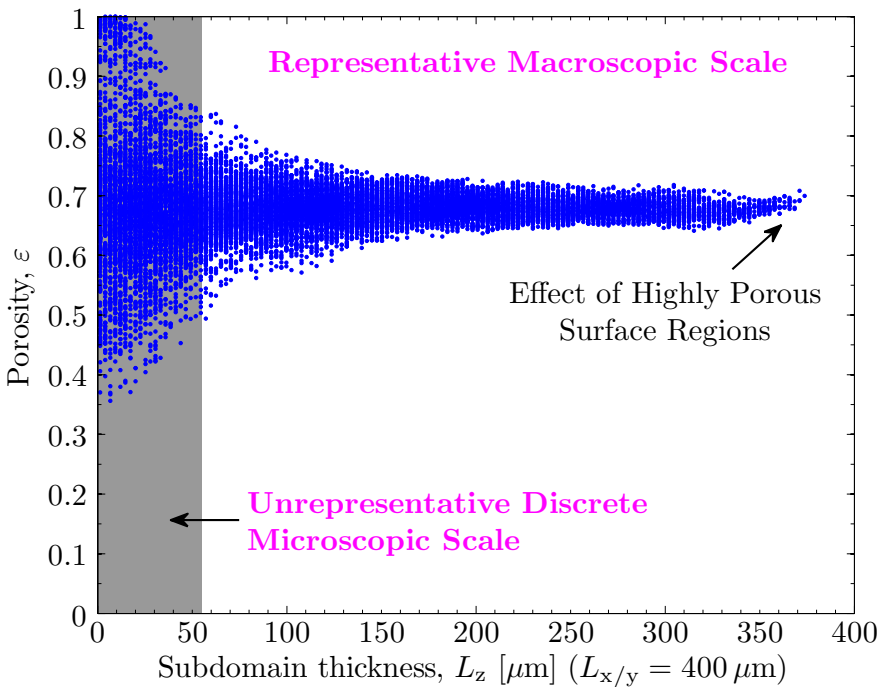

(c)

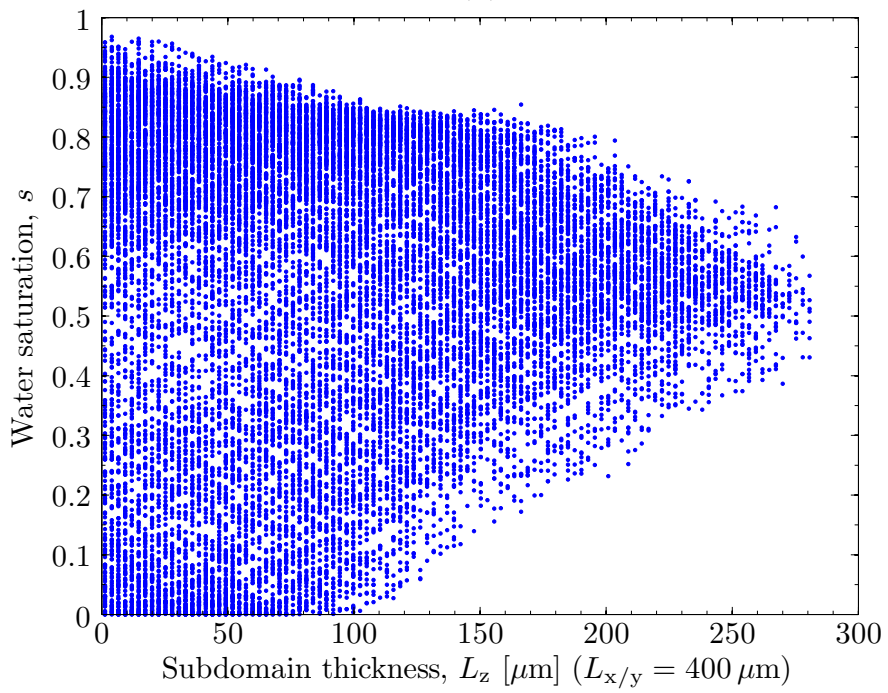


(b)
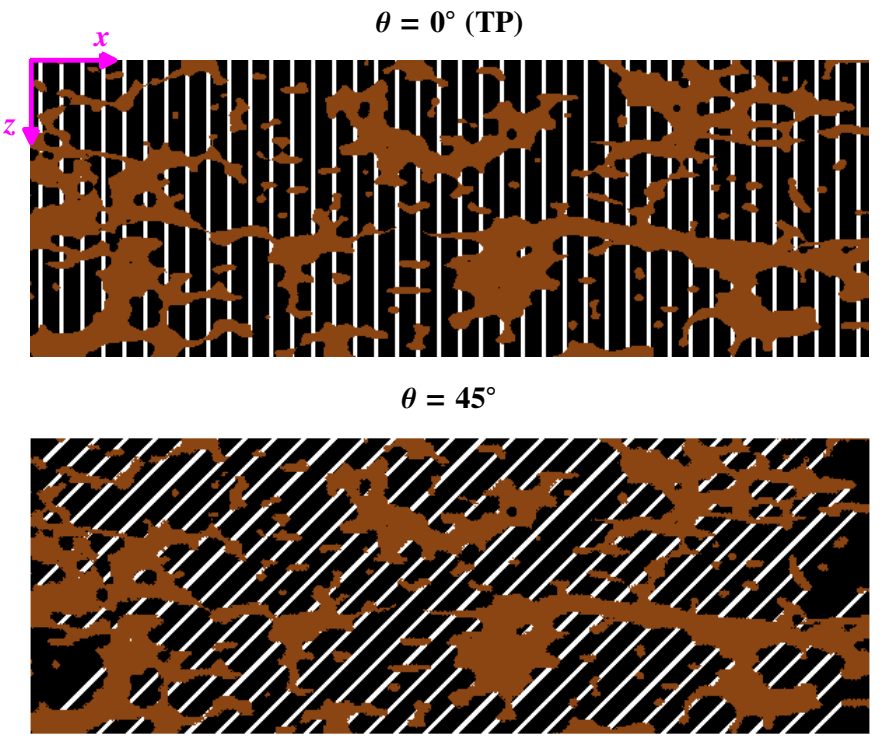

$\theta=90^{\circ}(\mathrm{IP})$

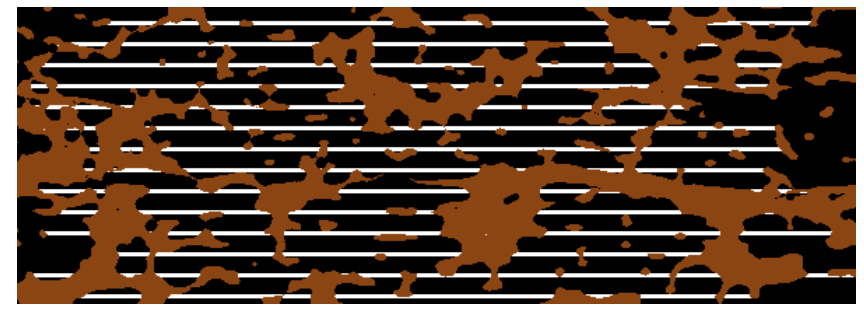

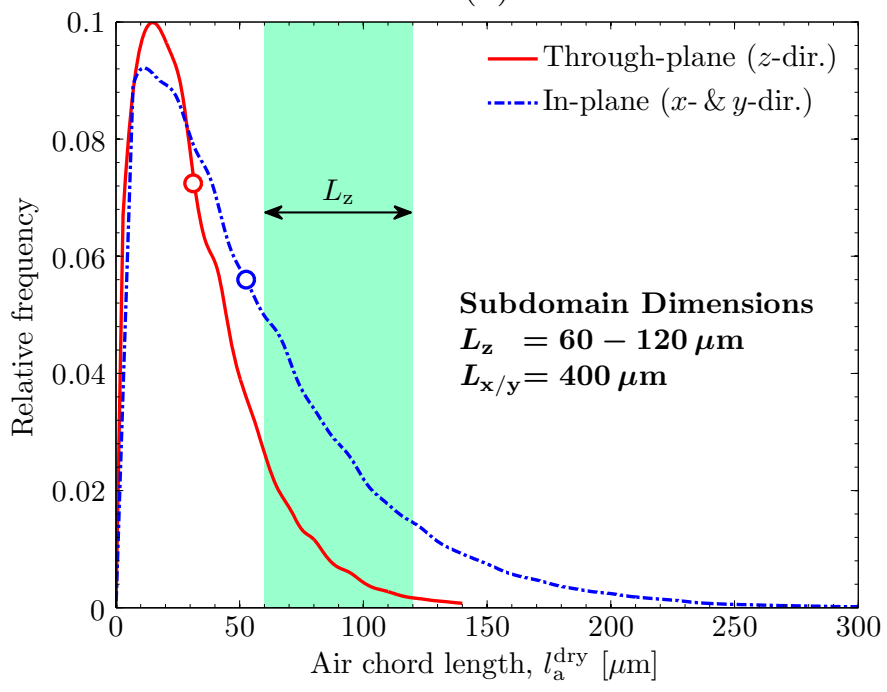

(c)
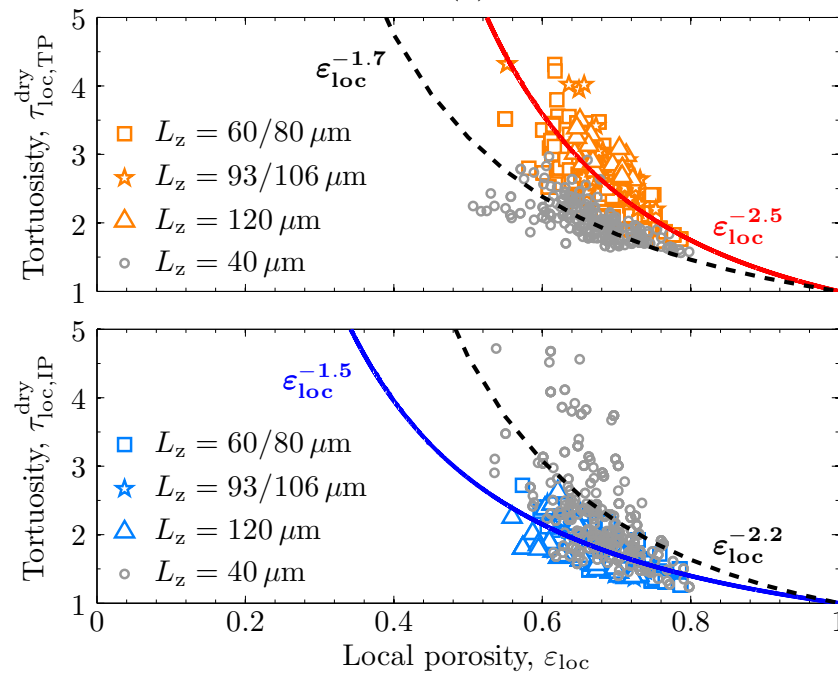
(a)

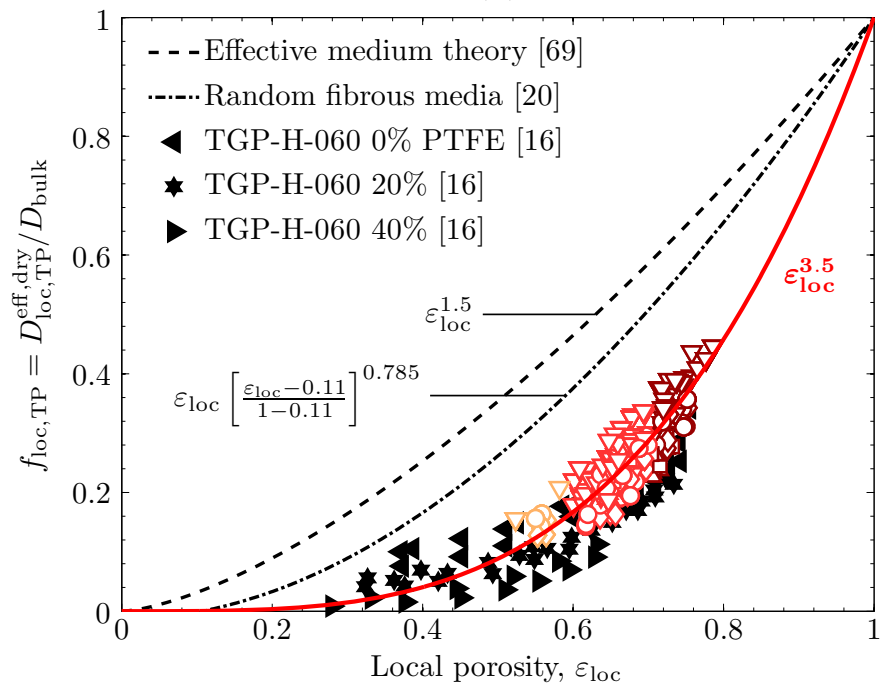

(c)

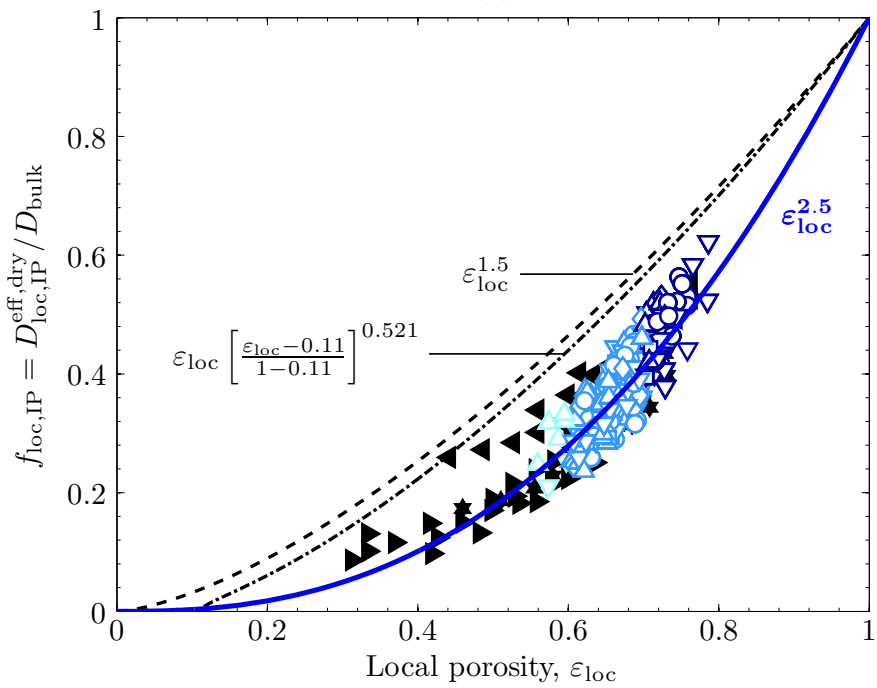

(b)

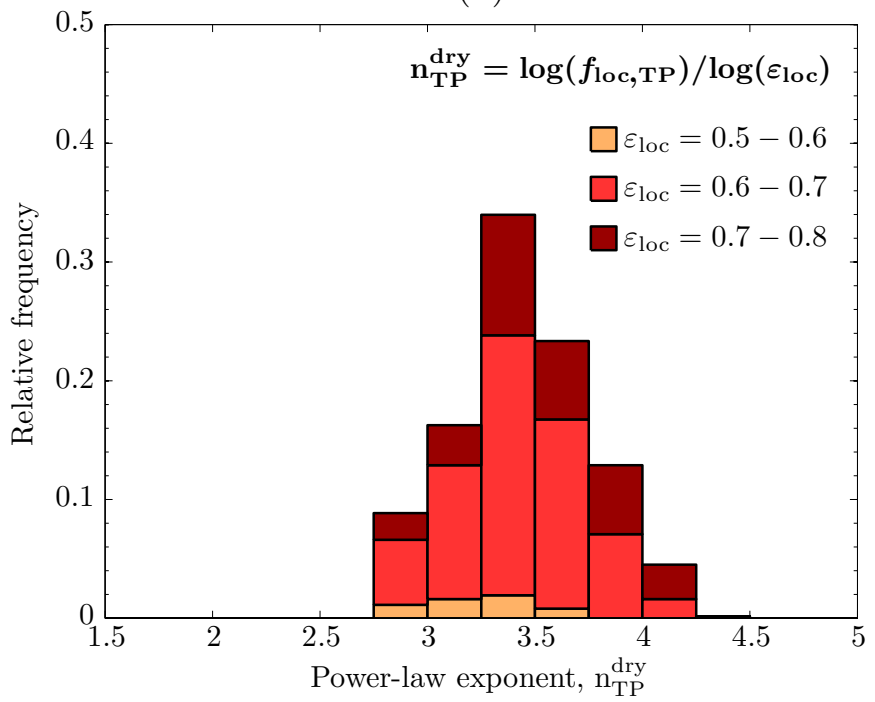

(d)

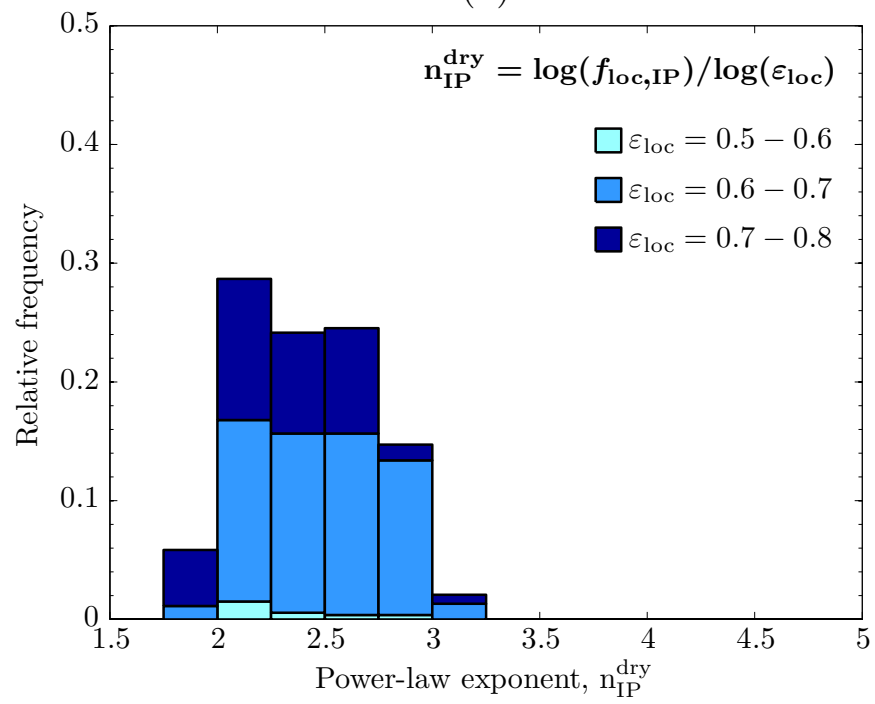

$\nabla$ Present work $\left(L_{\mathrm{z}}=60 \mu \mathrm{m}\right) \quad \bigcirc(80 \mu \mathrm{m}) \diamond(93 \mu \mathrm{m}) \quad \square(106 \mu \mathrm{m}) \quad \Delta(120 \mu \mathrm{m})$ 
(a)

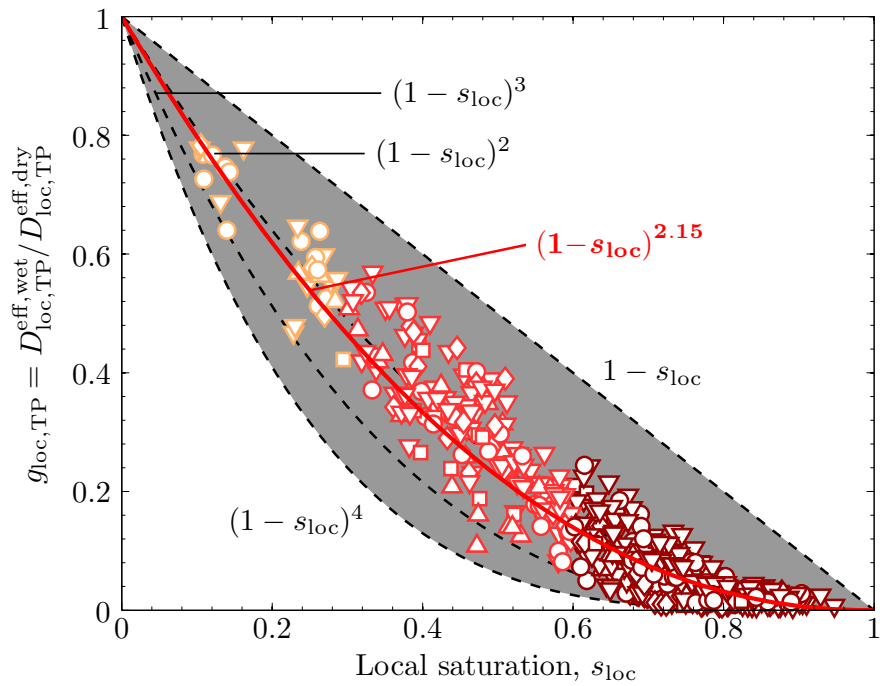

(c)

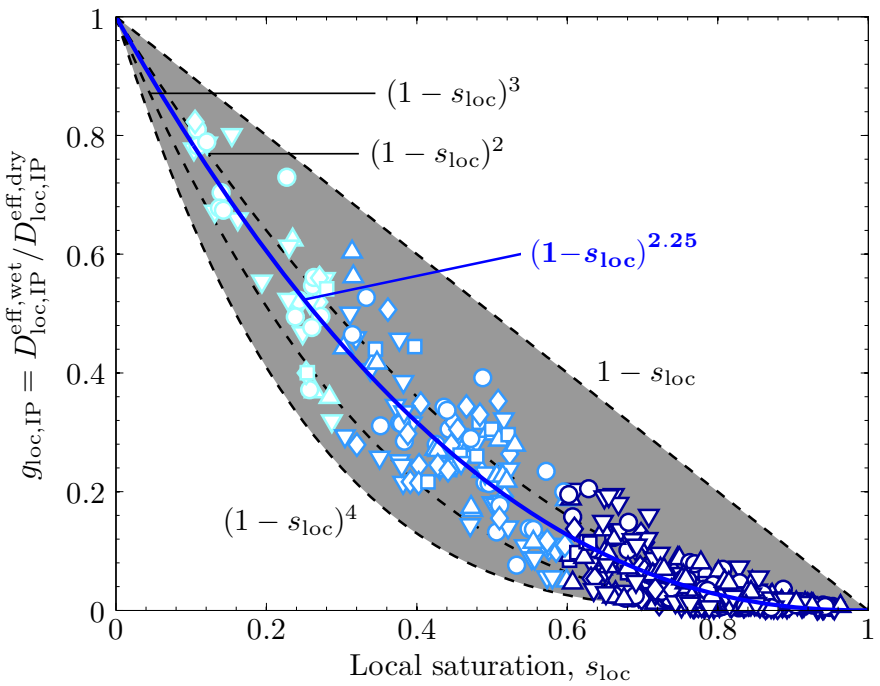

(b)

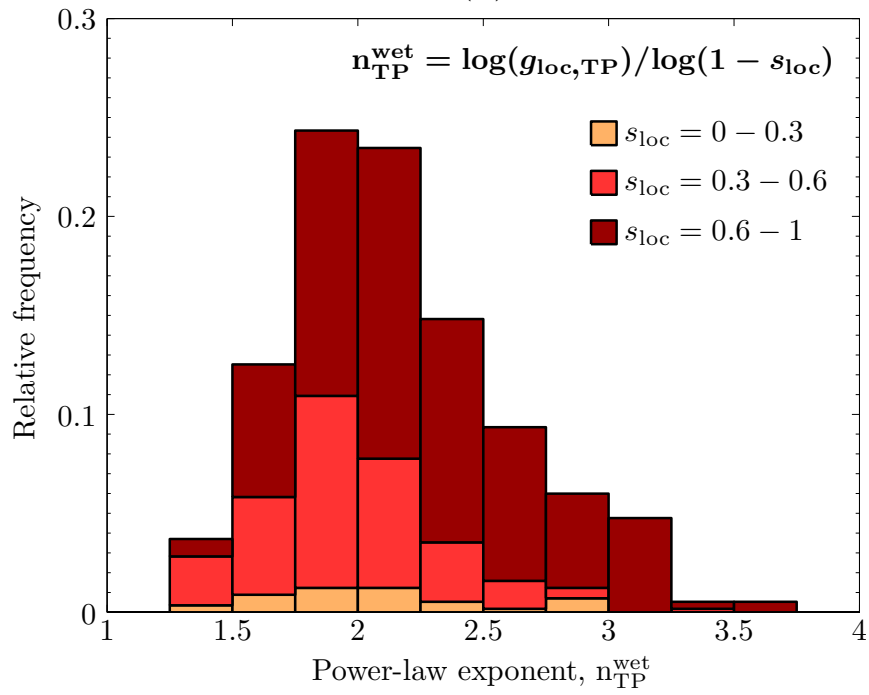

(d)

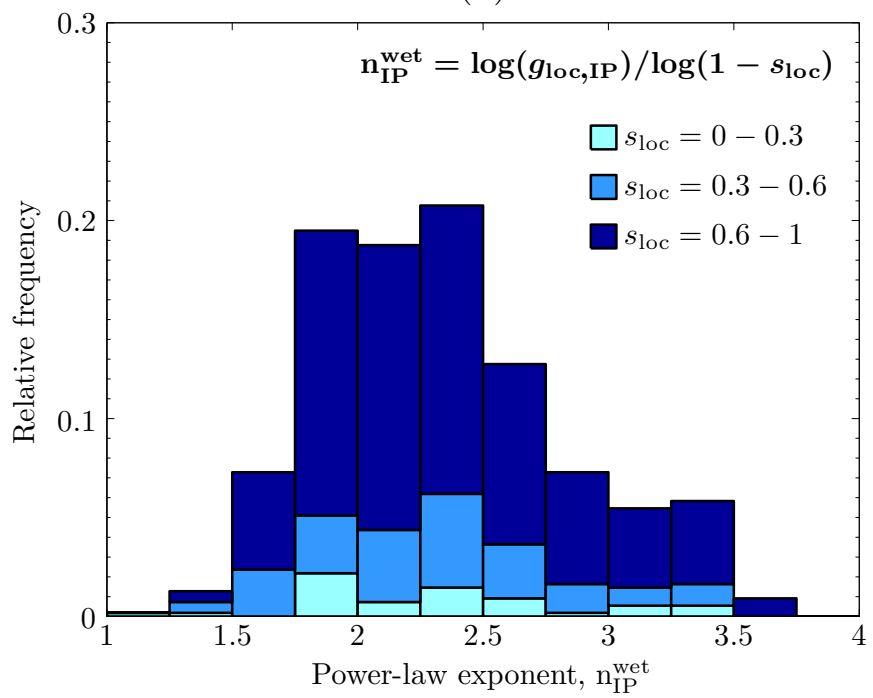

$\nabla$ Present work $\left(L_{\mathrm{z}}=60 \mu \mathrm{m}\right) \quad \bigcirc(80 \mu \mathrm{m}) \diamond(93 \mu \mathrm{m}) \quad \square(106 \mu \mathrm{m}) \quad \Delta(120 \mu \mathrm{m})$ 

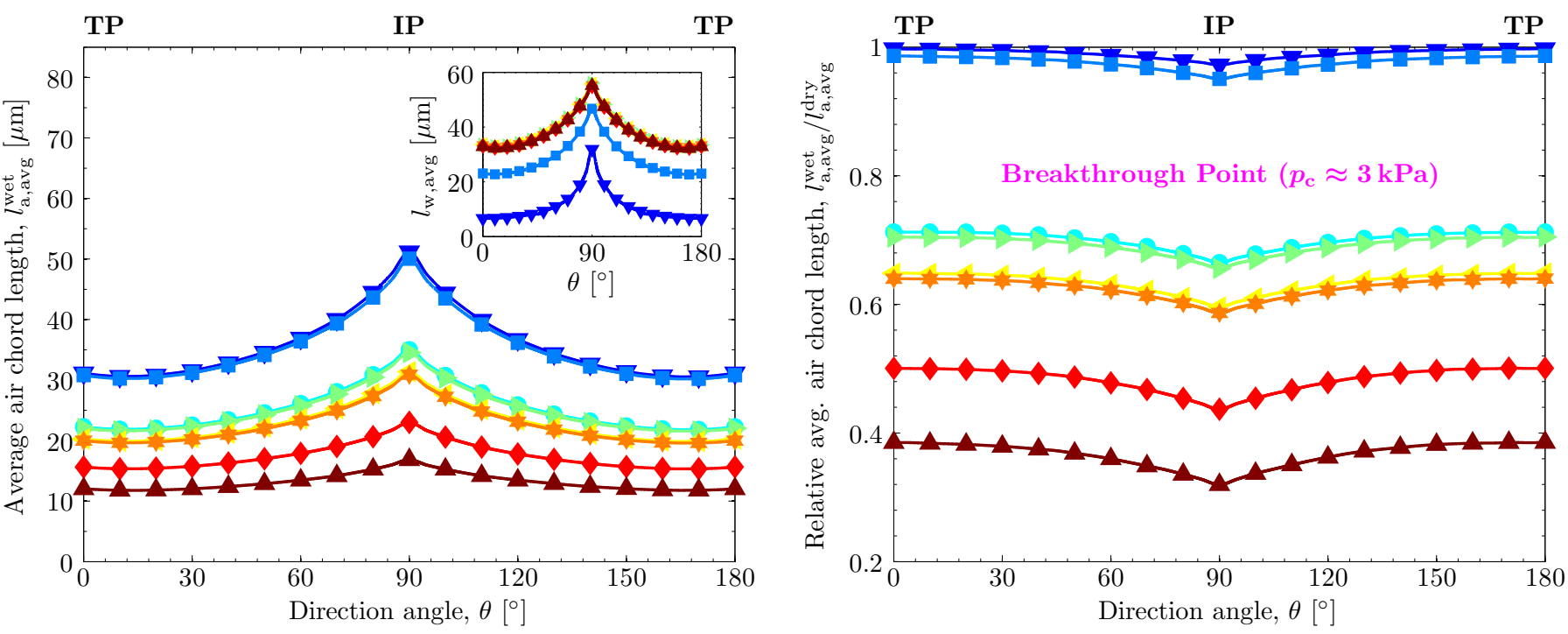

$\neg p_{\mathrm{c}}=1 \mathrm{kPa}\left(s_{\mathrm{avg}}=0.01\right)-2(0.11)-0-3(0.53)$

$4(0.54)$

$5(0.62) \multimap 6(0.63) \smile-7(0.79)$

$8(0.9)$ 
